\title{
Existence and Ulam-Hyers stability of a kind of fractional-order multiple point BVP involving noninstantaneous impulses and abstract bounded operator
}

\author{
Kaihong Zhao ${ }^{1 *}$ (D) and Shoukai Deng ${ }^{1}$
}

\author{
"Correspondence: \\ zhaokaihongs@126.com \\ 1 Department of Applied \\ Mathematics, Kunming University of \\ Science and Technology, Kunming, \\ Yunnan 650093, China
}

\begin{abstract}
In this paper, we mainly study a kind of fractional-order multiple point boundary value problem involving noninstantaneous impulse and abstract bounded operator. The existence and uniqueness is obtained by the Banach contraction principle. And by applying direct analysis methods, we establish some conditions of the Ulam-Hyers stability for this problem. Finally, an interesting application example is given to illustrate the validity of the results.
\end{abstract}

MSC: 34A08; 34B10; 34B37; 34D20

Keywords: Fractional-order multiple point BVP; Existence and Ulam-Hyers stability; Noninstantaneous impulses; Abstract bounded operator

\section{Introduction}

As a useful mathematical model, the multiple point boundary value problem of fractionalorder differential equation is used to describe many phenomena and processes such as in blood flow, chemical engineering, thermo-elasticity, underground water flow, population dynamics, and so on. The existence and attractivity of solutions for fractional differential boundary value problems is always a hot topic, and the latest research results can be found in the literature [1-6]. However, in practical applications, people pay more attention to whether a system is stable or not. So concepts and theories of stability such as those of Lyapunov, Lagrange, Poisson, Popov, and so forth, have been proposed. A kind of stability known as Ulam-Hyers stability has been put forward by Ulam and Hyers [7, 8]. This type of stability has been widely studied by many scholars since it was proposed. There have been many papers on this this stability (see [9-20]).

It is well known that impulse phenomenon is universal and inevitable in some fields of natural science, engineering technology, and even social science. The most important type of impulse is called the noninstantaneous impulse. This type of impulsive process is not instantaneous, but takes some time to complete. For example, in a similar process, drugs are absorbed, diffused, and metabolized in the human body. The noninstantaneous impulses

(c) The Author(s) 2021. This article is licensed under a Creative Commons Attribution 4.0 International License, which permits use, sharing, adaptation, distribution and reproduction in any medium or format, as long as you give appropriate credit to the original author(s) and the source, provide a link to the Creative Commons licence, and indicate if changes were made. The images or other third party material in this article are included in the article's Creative Commons licence, unless indicated otherwise in a credit line to the material. If material is not included in the article's Creative Commons licence and your intended use is not permitted by statutory regulation or exceeds the permitted use, you will need to obtain permission directly from the copyright holder. To view a copy of this licence, visit http://creativecommons.org/licenses/by/4.0/. 
are common in pharmacokinetics, agricultural pest control, sustainable fisheries production, and wildlife conservation. In the functional differential equation, the instantaneous pulse only changes the behavior of the solution of the equation at the point of the pulse, while the noninstantaneous pulse changes the behavior of the solution of the equation on a continuous interval. In contrast, the study of a noninstantaneous impulse functional differential equation is more difficult than that of a differential equation. In recent years, the study of noninstantaneous impulsive fractional order differential systems has attracted the attention of some researchers, and some good results have been obtained (see [2130]). However, relatively little research has been done on the fractional-order differential equation dynamics of noninstantaneous impulses.

Inspired by the above reasons, this paper mainly considers the following fractional-order multiple point boundary value problem involving noninstantaneous impulse and abstract bounded operator of the form:

$$
\left\{\begin{array}{l}
\left.{ }^{c} D_{s_{i}^{+}}^{\beta}{ }^{c} D_{s_{i}^{+}}^{\alpha}+\mathcal{A}\right) x(t)=f\left(t, x(t),{ }^{c} D_{s_{i}^{+}}^{\alpha} x(t)\right), \quad t \in\left(s_{i}, t_{i+1}\right] \subset J, i=0,1, \ldots, m, \\
x(t)=g_{i}\left(t, x\left(t_{i}^{-}\right)\right), \quad t \in\left(t_{i}, s_{i}\right] \subset J, i=1,2, \ldots, m, \\
x(0)=x(1)=x\left(\eta_{i+1}\right)=x\left(\zeta_{i+1}\right)=0, \quad \eta_{i+1}, \zeta_{i+1} \in\left(s_{i}, t_{i+1}\right) \subset J, i=0,1, \ldots, m,
\end{array}\right.
$$

where $J=[0,1], 0<\alpha<1,1<\beta<2,{ }^{c} D_{s_{i}^{+}}^{*}$ stands for the Caputo fractional derivative of order $* ; \mathcal{A} \in C^{2}(\mathbb{R}, \mathbb{R})$ is a bounded operator; $f \in C^{1}\left(J \times \mathbb{R}^{2}, \mathbb{R}\right), g_{i} \in C\left(\left[t_{i}, s_{i}\right] \times \mathbb{R}, \mathbb{R}\right)$ are called noninstantaneous impulsive functions, for all $i=1,2, \ldots, m$. The impulsive points and boundary points satisfy $0=s_{0}<\eta_{1}<\zeta_{1}<t_{1}<s_{1}<\eta_{2}<\zeta_{2}<t_{2}<\cdots<t_{m}<s_{m}<\eta_{m}<$ $\zeta_{m}<t_{m+1}=1$.

Furthermore, the investigation of (1.1) was also motivated by the work of Zada et al. [31]. They discussed nonlinear implicit fractional differential equations with noninstantaneous integral impulses as follows:

$$
\left\{\begin{array}{l}
{ }^{c} D_{0, t}^{\beta} y(t)=f\left(t, y(t),{ }^{c} D_{0, t}^{\beta} y(t)\right), \quad t \in\left(t_{k}, s_{k}\right] \subset J, k=0,1, \ldots, m, 0<\beta \leq 1, \\
y(t)=I_{s_{k-1}, t_{k}}^{\beta} \xi_{k}(t, y(t)), \quad t \in\left(s_{k-1}, t_{k}\right] \subset J, k=1,2, \ldots, m, \\
y(0)=I_{0, T}^{\beta} \eta(t, y(t)),
\end{array}\right.
$$

where $T>0, J=[0, T], 0=t_{0}<s_{0}<t_{1}<s_{1}<\cdots<t_{m}=T,{ }^{c} D_{0, t}^{\beta}$ stands the Caputo fractional derivative of order $\beta ; f \in C\left(J \times \mathbb{R}^{2}, \mathbb{R}\right), \eta \in C([0, T] \times \mathbb{R}, \mathbb{R}), \xi_{k} \in C\left(\left[s_{k-1}, t_{k}\right] \times \mathbb{R}, \mathbb{R}\right)$ are noninstantaneous impulsive functions, for all $i=1,2, \ldots, m ; I_{s_{k-1}, t_{k}}^{\beta}$ and $I_{0, T}^{\beta}$ are given to fractional integrals, respectively. By the generalized Diaz-Margolis's fixed point theorem, the authors obtained Ulam-Hyers, Ulam-Hyers-Rassias, and generalized Ulam-HyersRassias stability for this problem.

Our aim is study the existence, uniqueness, and Ulam-type stability for problem (1.1) in this paper. The rest of the paper is organized as follows. Section 2 contains some useful notations and lemmas. The main results are proved in Sect. 3. In Sect. 5, an example is given to illustrate our main results. Finally, we conclude with a discussion of the importance of the studied problem (1.1) and summarize our obtained results in Sect. 5.

\section{Preliminaries}

In this section, we introduce some necessary definitions and lemmas of fractional calculus and present preliminary results. At the same time, we need to define some Banach 
spaces. Let $C(J, \mathbb{R})$ be the Banach space of all continuous functions from $J$ into $\mathbb{R}$ with the norm $\|x\|_{C}=\sup _{t \in J}|x(t)|$. To discuss the existence and Ulam-Hyers stability of solutions for problem (1.1), we need to introduce the space $X=\operatorname{PC}^{3}(J, \mathbb{R})$ of piecewise continuous functions

$$
\begin{aligned}
X=\mathrm{PC}^{3}(J, \mathbb{R})= & \left\{x \in C^{3}(J, \mathbb{R}): x \in C\left(\left(t_{i}, s_{i}\right], \mathbb{R}\right), x\left(t_{i}^{-}\right) \text {and } x\left(s_{i}^{+}\right)\right. \\
& \text {exist with } \left.x\left(s_{i}^{+}\right)=x\left(s_{i}\right), i=1, \ldots, m\right\} .
\end{aligned}
$$

Clearly, $X=\mathrm{PC}^{3}(J, \mathbb{R})$ is a Banach spaces equipped with the norm

$$
\|x\|_{X}=\max \left\{\sup _{t \in J}|x(t)|, \max _{0 \leq i \leq m} \sup _{s_{i}<t \leq t_{i+1}}\left|{ }^{c} D_{s_{i}^{+}}^{\alpha} x(t)\right|\right\} .
$$

Definition 2.1 ([32]) The Riemann-Liouville fractional integral of order $\alpha>0$ of a continuous function $f:[0, \infty) \rightarrow R$ is defined by

$$
I_{0^{+}}^{\alpha} f(t)=\frac{1}{\Gamma(\alpha)} \int_{0}^{t}(t-s)^{\alpha-1} f(s) d s
$$

provided that the right-hand side is pointwise defined on $[0, \infty)$.

Definition 2.2 ([32]) The Riemann-Liouville fractional derivative of order $\alpha>0$ of a continuous function $f:[0, \infty) \rightarrow R$ is defined by

$$
{ }^{L} D_{0^{+}}^{\alpha} f(t)=\frac{1}{\Gamma(n-\alpha)} \frac{d^{n}}{d t^{n}} \int_{0}^{t}(t-s)^{n-\alpha-1} f(s) d s,
$$

where $n-1<\alpha \leq n$, provided that the right-hand side is pointwise defined on $[0, \infty)$.

Definition 2.3 ([32]) If $f \in C^{n}[0, \infty)$ and $\alpha>0$, then the Caputo fractional derivative of order $\alpha$ is given by

$$
{ }^{c} D_{0^{+}}^{\alpha} f(t)=\frac{1}{\Gamma(n-\alpha)} \int_{0}^{t}(t-s)^{n-\alpha-1} f^{(n)}(s) d s,
$$

where $n-1<\alpha \leq n$, provided that the right-hand side is pointwise defined on $[0, \infty)$.

Lemma 2.1 ([9]) If $u \in C^{n}[0,1]$ and $n-1<p \leq n$, then

$$
I_{0+}^{p}\left({ }^{c} D_{0+}^{p} u(t)\right)=u(t)-\sum_{k=0}^{n-1} \frac{u^{(k)}(0)}{k !} t^{k} .
$$

To investigate the existence and Ulam-Hyers stability of solutions for the problem (1.1), we need to consider the impulsive integral nonlinear equation (2.1) as follows:

$$
x(t)= \begin{cases}F_{0}(t)-\frac{t^{\alpha+1}-t^{\alpha} \eta_{1}}{\zeta_{1}^{\alpha+1}-\zeta_{1}^{\alpha} \eta_{1}} F_{0}\left(\zeta_{1}\right)-\frac{t^{\alpha} \zeta_{1}-t^{\alpha+1}}{\zeta_{1} \eta_{1}^{\alpha}-\eta_{1}^{\alpha+1}} F_{0}\left(\eta_{1}\right), & t \in\left[0, t_{1}\right], \\ g_{i}\left(t, x\left(s_{i}^{-}\right)\right), & t \in\left(t_{i}, s_{i}\right], i=1,2, \ldots, m, \\ F_{i}(t)-c_{i 1}^{*} t^{\alpha+1}-c_{i 2}^{*} t^{\alpha}-c_{i 3}^{*}, & t \in\left(s_{i}, t_{i+1}\right], i=1,2, \ldots, m,\end{cases}
$$


where

$$
\begin{aligned}
F_{i}(t)= & \int_{s_{i}}^{t} \frac{(t-u)^{\alpha-1}}{\Gamma(\alpha)}\left(\int_{s_{i}}^{u} \frac{(u-s)^{\beta-1}}{\Gamma(\beta)} f\left(s, x(s),{ }^{c} D_{s_{i}^{+}}^{\alpha} x(s)\right) d s-(\mathcal{A} x)(u)\right) d u \\
= & I_{s_{i}^{+}}^{\alpha}\left[I_{s_{i}^{+}}^{\beta} f\left(u, x(u),{ }^{c} D_{s_{i}^{+}}^{\alpha} x(u)\right)-(\mathcal{A} x)(u)\right](t), \\
\mathcal{M}_{i}= & \frac{1}{\left(\eta_{i+1}^{\alpha+1}-s_{i}^{\alpha+1}\right)\left(\zeta_{i+1}^{\alpha}-s_{i}^{\alpha}\right)-\left(\zeta_{i+1}^{\alpha}-s_{i}^{\alpha}\right)\left(\eta_{i+1}^{\alpha}-s_{i}^{\alpha}\right)}, \\
c_{i 1}^{*}= & \mathcal{M}_{i}\left[\left(\zeta_{i+1}^{\alpha}-s_{i}^{\alpha}\right) F_{i}\left(\eta_{i+1}\right)-\left(\eta_{i+1}^{\alpha}-s_{i}^{\alpha}\right) F_{i}\left(\zeta_{i+1}\right)-\left(\zeta_{i+1}^{\alpha}-\eta_{i+1}^{\alpha}\right) g_{i}\left(s_{i}, x\left(t_{i}^{-}\right)\right)\right], \\
c_{i 2}^{*}= & \mathcal{M}_{i}\left[\left(\eta_{i+1}^{\alpha+1}-s_{i}^{\alpha+1}\right) F_{i}\left(\zeta_{i+1}\right)-\left(\zeta_{i+1}^{\alpha+1}-s_{i}^{\alpha+1}\right) F_{i}\left(\eta_{i+1}\right)+\left(\zeta_{i+1}^{\alpha+1}-\eta_{i+1}^{\alpha+1}\right) g_{i}\left(s_{i}, x\left(t_{i}^{-}\right)\right)\right], \\
c_{i 3}^{*}= & \mathcal{M}_{i}\left[\left(\zeta_{i+1}^{\alpha+1} s_{i}^{\alpha}-s_{i}^{\alpha+1} \zeta_{i+1}^{\alpha}\right) F_{i}\left(\eta_{i+1}\right)-\left(\eta_{i+1}^{\alpha+1} s_{i}^{\alpha}-s_{i}^{\alpha+1} \eta_{i+1}^{\alpha}\right) F_{i}\left(\zeta_{i+1}\right)\right. \\
& \left.-\left(\zeta_{i+1}^{\alpha+1} \eta_{i+1}^{\alpha}-\eta_{i+1}^{\alpha+1} \zeta_{i+1}^{\alpha}\right) g_{i}\left(s_{i}, x\left(t_{i}^{-}\right)\right)\right] .
\end{aligned}
$$

Lemma 2.2 Assume that $\mathcal{A} \in C^{2}(\mathbb{R}, \mathbb{R})$ is a bounded operator, and $f \in C^{1}\left(J \times \mathbb{R}^{2}, \mathbb{R}\right)$. Then $x(t) \in X$ is a solution of the problem (1.1) if and only if $x(t) \in X$ is also a solution of the integral equation (2.1).

Proof Assume that $x(t) \in X$ is a known solution of (1.1). When $t \in\left[s_{0}, t_{1}\right]=\left[0, t_{1}\right]$, according to Lemma 2.1, we have

$$
\begin{aligned}
x(t)= & \int_{0}^{t} \frac{(t-u)^{\alpha-1}}{\Gamma(\alpha)}\left(\int_{0}^{u} \frac{(u-s)^{\beta-1}}{\Gamma(\beta)} f\left(s, x(s),{ }^{c} D_{0^{+}}^{\alpha} x(s)\right) d s-(\mathcal{A} x)(u)\right) d u \\
& -c_{01} \frac{t^{\alpha+1}}{\Gamma(\alpha+2)}-c_{02} \frac{t^{\alpha}}{\Gamma(\alpha+1)}-c_{03} .
\end{aligned}
$$

Using the conditions $x(0)=x\left(\eta_{1}\right)=x\left(\zeta_{1}\right)=0$, we obtain

$$
\begin{aligned}
& c_{01}=\Gamma(\alpha+2) \frac{\eta_{1}^{\alpha} F_{0}\left(\zeta_{1}\right)-\zeta_{1}^{\alpha} F_{0}\left(\eta_{1}\right)}{\zeta_{1}^{\alpha+1} \eta_{1}^{\alpha}-\zeta_{1}^{\alpha} \eta_{1}^{\alpha+1}}, \quad c_{02}=\Gamma(\alpha+1) \frac{\zeta_{1}^{\alpha+1} F_{0}\left(\eta_{1}\right)-\eta_{1}^{\alpha+1} F_{0}\left(\zeta_{1}\right)}{\zeta_{1}^{\alpha+1} \eta_{1}^{\alpha}-\zeta_{1}^{\alpha} \eta_{1}^{\alpha+1}}, \\
& c_{03}=0 .
\end{aligned}
$$

Substituting $c_{01}, c_{02}$, and $c_{03}$ into (2.2), we get

$$
x(t)=F_{0}(t)-\frac{t^{\alpha+1}-t^{\alpha} \eta_{1}}{\zeta_{1}^{\alpha+1}-\zeta_{1}^{\alpha} \eta_{1}} F_{0}\left(\zeta_{1}\right)-\frac{t^{\alpha} \zeta_{1}-t^{\alpha+1}}{\zeta_{1} \eta_{1}^{\alpha}-\eta_{1}^{\alpha+1}} F_{0}\left(\eta_{1}\right)
$$

When $t \in\left(t_{1}, s_{1}\right]$, it follows from (2.1) that

$$
x(t)=g_{1}\left(t, x\left(t_{1}^{-}\right)\right)
$$

When $t \in\left(s_{1}, t_{2}\right]$, from Lemma 2.1, we have

$$
\begin{aligned}
x(t)= & \int_{s_{1}}^{t} \frac{(t-u)^{\alpha-1}}{\Gamma(\alpha)}\left(\int_{s_{1}}^{u} \frac{(u-s)^{\beta-1}}{\Gamma(\beta)} f\left(s, x(s),{ }^{c} D_{s_{1}^{+}}^{\alpha} x(s)\right) d s-(\mathcal{A} x)(u)\right) d u \\
& -c_{11} \frac{t^{\alpha+1}}{\Gamma(\alpha+2)}-c_{12} \frac{t^{\alpha}}{\Gamma(\alpha+1)}-c_{13} .
\end{aligned}
$$


In view the conditions $x\left(s_{1}\right)=x\left(s_{1}^{+}\right)$and $x\left(\zeta_{2}\right)=x\left(\eta_{2}\right)=0$, we derive that

$$
\left\{\begin{array}{l}
c_{11} \frac{s_{1}^{\alpha+1}}{\Gamma(\alpha+2)}+c_{12} \frac{s_{1}^{\alpha}}{\Gamma(\alpha+1)}+c_{13}=g_{1}\left(s_{1}, x\left(t_{1}^{-}\right)\right) \\
c_{11} \frac{\eta_{2}^{\alpha+1}}{\Gamma(\alpha+2)}+c_{12} \frac{\eta_{2}^{\alpha}}{\Gamma(\alpha+1)}+c_{13}=F_{1}\left(\eta_{2}\right) \\
c_{11} \frac{\zeta_{2}^{\alpha+1}}{\Gamma(\alpha+2)}+c_{12} \frac{\zeta_{2}^{\alpha}}{\Gamma(\alpha+1)}+c_{13}=F_{1}\left(\zeta_{2}\right)
\end{array}\right.
$$

which implies that

$$
\begin{aligned}
c_{11}= & \Gamma(\alpha+2) \mathcal{M}_{1}\left[( \zeta _ { 2 } ^ { \alpha } - s _ { 1 } ^ { \alpha } ) \left(F_{1}\left(\eta_{2}\right)-g_{1}\left(s_{1}, x\left(t_{1}^{-}\right)\right)\right.\right. \\
& -\left(\eta_{2}^{\alpha}-s_{1}^{\alpha}\right)\left(F_{1}\left(\zeta_{2}\right)-g_{1}\left(s_{1}, x\left(t_{1}^{-}\right)\right)\right], \\
c_{12}= & \Gamma(\alpha+1) \mathcal{M}_{1}\left[( \eta _ { 2 } ^ { \alpha + 1 } - s _ { 1 } ^ { \alpha + 1 } ) \left(F_{1}\left(\zeta_{2}\right)-g_{1}\left(s_{1}, x\left(t_{1}^{-}\right)\right)\right.\right. \\
& -\left(\zeta_{2}^{\alpha+1}-s_{1}^{\alpha+1}\right)\left(F_{1}\left(\eta_{2}\right)-g_{1}\left(s_{1}, x\left(t_{1}^{-}\right)\right)\right], \\
c_{13}= & \mathcal{M}_{1}\left[\left(\zeta_{2}^{\alpha+1} s_{1}^{\alpha}-s_{1}^{\alpha+1} \zeta_{2}^{\alpha}\right) F_{1}\left(\eta_{2}\right)-\left(\eta_{2}^{\alpha+1} s_{1}^{\alpha}-s_{1}^{\alpha+1} \eta_{2}^{\alpha}\right) F_{1}\left(\zeta_{2}\right)\right. \\
& \left.-\left(\zeta_{2}^{\alpha+1} \eta_{2}^{\alpha}-\eta_{2}^{\alpha+1} \zeta_{2}^{\alpha}\right) g_{1}\left(s_{1}, x\left(t_{1}^{-}\right)\right)\right] .
\end{aligned}
$$

Substituting $c_{11}, c_{12}$, and $c_{13}$ into (2.3), we get

$$
x(t)=F_{1}(t)-c_{11}^{*} t^{\alpha+1}-c_{12}^{*} t^{\alpha}-c_{13}^{*},
$$

where

$$
\begin{aligned}
c_{11}^{*}= & \mathcal{M}_{1}\left[\left(\zeta_{2}^{\alpha}-s_{1}^{\alpha}\right) F_{1}\left(\eta_{2}\right)-\left(\eta_{2}^{\alpha}-s_{1}^{\alpha}\right) F_{1}\left(\zeta_{2}\right)-\left(\zeta_{2}^{\alpha}-\eta_{2}^{\alpha}\right) g_{1}\left(s_{1}, x\left(t_{1}^{-}\right)\right)\right], \\
c_{12}^{*}= & \mathcal{M}_{1}\left[\left(\eta_{2}^{\alpha+1}-s_{1}^{\alpha+1}\right) F_{1}\left(\zeta_{2}\right)-\left(\zeta_{2}^{\alpha+1}-s_{1}^{\alpha+1}\right) F_{1}\left(\eta_{2}\right)+\left(\zeta_{2}^{\alpha+1}-\eta_{2}^{\alpha+1}\right) g_{1}\left(s_{1}, x\left(t_{1}^{-}\right)\right)\right], \\
c_{13}^{*}= & \mathcal{M}_{1}\left[\left(\zeta_{2}^{\alpha+1} s_{1}^{\alpha}-s_{1}^{\alpha+1} \zeta_{2}^{\alpha}\right) F_{1}\left(\eta_{2}\right)-\left(\eta_{2}^{\alpha+1} s_{1}^{\alpha}-s_{1}^{\alpha+1} \eta_{2}^{\alpha}\right) F_{1}\left(\zeta_{2}\right)\right. \\
& \left.-\left(\zeta_{2}^{\alpha+1} \eta_{2}^{\alpha}-\eta_{2}^{\alpha+1} \zeta_{2}^{\alpha}\right) g_{1}\left(s_{1}, x\left(t_{1}^{-}\right)\right)\right] .
\end{aligned}
$$

Repeating the above arguments, we obtain

$$
\begin{aligned}
& x(t)=g_{i}\left(t, x\left(t_{i}^{-}\right)\right), \quad t \in\left(t_{i}, s_{i}\right], i=2,3, \ldots, m, \\
& x(t)=F_{i}(t)-c_{i 1}^{*} t^{\alpha+1}-c_{i 2}^{*} t^{\alpha}-c_{i 3}^{*}, \quad t \in\left(s_{i}, t_{i+1}\right], i=2,3, \ldots, m .
\end{aligned}
$$

Thus $x(t) \in X$ satisfies (2.1).

Now we shall show that if $x(t) \in X$ is a solution of (2.1), then $x(t) \in X$ is also a solution of (1.1). In fact, let $x(t) \in X$ be a solution of (2.1). When $t \in\left[0, t_{1}\right]$, taking the Caputo fractional derivative of order $\alpha$ on both sides of the first equation of (2.1), we obtain

$$
\begin{aligned}
{ }^{c} D_{0^{+}}^{\alpha} x(t)= & { }^{c} D_{0^{+}}^{\alpha} F_{0}(t)-\frac{F_{0}\left(\zeta_{1}\right)}{\zeta_{1}^{\alpha+1}-\zeta_{1}^{\alpha} \eta_{1}}{ }^{c} D_{0^{+}}^{\alpha}\left(t^{\alpha+1}-t^{\alpha} \eta_{1}\right)-\frac{F_{0}\left(\eta_{1}\right)}{\zeta_{1} \eta_{1}^{\alpha}-\eta_{1}^{\alpha+1}}{ }^{c} D_{0^{+}}^{\alpha}\left(t^{\alpha} \zeta_{1}-t^{\alpha+1}\right) \\
= & I_{0^{+}}^{\beta} f\left(t, x(t),{ }^{c} D_{0^{+}}^{\alpha} x(t)\right)-(\mathcal{A} x)(t)-\frac{F_{0}\left(\zeta_{1}\right)}{\zeta_{1}^{\alpha+1}-\zeta_{1}^{\alpha} \eta_{1}}\left(\Gamma(\alpha+2)-\Gamma(\alpha+1) \eta_{1}\right) \\
& -\frac{F_{0}\left(\eta_{1}\right)}{\zeta_{1} \eta_{1}^{\alpha}-\eta_{1}^{\alpha+1}}\left(\Gamma(\alpha+1) \zeta_{1}-\Gamma(\alpha+2)\right)
\end{aligned}
$$


which implies that

$$
\begin{aligned}
{ }^{c} D_{0^{+}}^{\alpha} x(t)+(\mathcal{A} x)(t)= & I_{0^{+}}^{\beta} f\left(t, x(t),{ }^{c} D_{0^{+}}^{\alpha} x(t)\right)-\frac{F_{0}\left(\zeta_{1}\right)}{\zeta_{1}^{\alpha+1}-\zeta_{1}^{\alpha} \eta_{1}}\left(\Gamma(\alpha+2)-\Gamma(\alpha+1) \eta_{1}\right) \\
& -\frac{F_{0}\left(\eta_{1}\right)}{\zeta_{1} \eta_{1}^{\alpha}-\eta_{1}^{\alpha+1}}\left(\Gamma(\alpha+1) \zeta_{1}-\Gamma(\alpha+2)\right) .
\end{aligned}
$$

Computing the Caputo fractional derivative of order $\beta$ on both sides of (2.5), we derive

$$
{ }^{c} D_{0^{+}}^{\beta}\left({ }^{c} D_{0^{+}}^{\alpha} x(t)+(\mathcal{A} x)(t)\right)=f\left(t, x(t),{ }^{c} D_{0^{+}}^{\alpha} x(t)\right),
$$

that is,

$$
{ }^{c} D_{0^{+}}^{\beta}\left({ }^{c} D_{0^{+}}^{\alpha}+(\mathcal{A}) x(t)\right)=f\left(t, x(t),{ }^{c} D_{0^{+}}^{\alpha} x(t)\right) .
$$

It follows from the first equation of (2.1) that

$$
x(0)=x\left(\eta_{1}\right)=x\left(\zeta_{1}\right)=0 .
$$

When $t \in\left(s_{i}, t_{i+1}\right], i=1,2, \ldots, m$, by employing similar arguments, as those used to obtain (2.4)-(2.7), on the third equation of (2.1), we get

$$
\begin{aligned}
& { }^{c} D_{s_{i}^{+}}^{\beta}\left({ }^{c} D_{s_{i}^{+}}^{\alpha}+\mathcal{A}\right) x(t)=f\left(t, x(t),{ }^{c} D_{s_{i}^{+}}^{\alpha} x(t)\right), \\
& x\left(\eta_{i}\right)=x\left(\zeta_{i}\right)=0 .
\end{aligned}
$$

When $t \in\left(t_{i}, s_{i}\right], i=1,2, \ldots, m$, we notice that the second equation of (1.1) and (2.1) have the same expression. Thus we verify that $x(t) \in X$ also satisfies (1.1). The proof is completed.

Next we state the Ulam-Hyers stability concept and some facts.

Let $y \in X$ and $\epsilon>0$. Consider the following inequality:

$$
\left\{\begin{array}{l}
\left|{ }^{c} D_{s_{i}^{+}}^{\beta}\left({ }^{c} D_{s_{i}^{+}}^{\alpha}+\mathcal{A}\right) y(t)-f\left(t, y(t),{ }^{c} D_{s_{i}^{+}}^{\alpha} y(t)\right)\right| \leq \epsilon, \quad t \in\left(s_{i}, t_{i+1}\right], \\
\left|y(t)-g_{i}\left(t, y\left(t_{i}^{-}\right)\right)\right| \leq \epsilon, \quad t \in\left(t_{i}, s_{i}\right] .
\end{array}\right.
$$

Definition 2.4 The problem (1.1) is Ulam-Hyers stable if for a given $\varepsilon>0$ and for each solution $y \in X$ of the inequality (2.10), there exist a constant $c_{f, \alpha, \beta, g_{i}}>0$ and a solution $x \in X$ of the problem (1.1) such that

$$
|y(t)-x(t)| \leq c_{f, \alpha, \beta, g_{i}} \varepsilon
$$

Remark 2.1 Assume that $\mathcal{A} \in C^{2}(\mathbb{R}, \mathbb{R})$ is a bounded operator, and $f \in C^{1}\left(J \times \mathbb{R}^{2}, \mathbb{R}\right)$. A function $y \in X$ is a solution of the inequality (2.10) if and only if there exist a function $\mathcal{B}(t) \in X$ and a sequence $\left\{\mathcal{B}_{i}\right\}_{i=1}^{m}$ such that

(i) $|\mathcal{B}(t)| \leq \varepsilon, t \in J$ and $\left|\mathcal{B}_{i}\right| \leq \varepsilon, i=1,2, \ldots, m$;

(ii) ${ }^{c} D_{s_{i}^{+}}^{\beta}\left({ }^{c} D_{s_{i}^{+}}^{\alpha}+\mathcal{A}\right) y(t)=f\left(t, y(t),{ }^{c} D_{s_{i}^{+}}^{\alpha} y(t)\right)+\mathcal{B}(t)$;

(iii) $y(t)=g_{i}\left(t, y\left(t_{i}^{-}\right)\right)+\mathcal{B}_{i}, i=1,2, \ldots, m$. 
Lemma 2.3 Assume that $\mathcal{A} \in C^{2}(\mathbb{R}, \mathbb{R})$ is a bounded operator, and $f \in C^{1}\left(J \times \mathbb{R}^{2}, \mathbb{R}\right)$. If the function $y \in X$ is a solution of inequality (2.10), then $y$ is a solution of the following inequality:

$$
\left\{\begin{aligned}
\mid y(t) & -F_{0}(t)+\frac{t^{\alpha+1}-t^{\alpha} \eta_{1}}{\zeta_{1}^{\alpha+1}-\zeta_{1}^{\alpha} \eta_{1}} F_{0}\left(\zeta_{1}\right)+\frac{t^{\alpha} \zeta_{1}-t^{\alpha+1}}{\zeta_{1} \eta_{1}^{\alpha}-\eta_{1}^{\alpha+1}} F_{0}\left(\eta_{1}\right) \mid \\
\leq & \frac{\varepsilon}{\Gamma(\alpha+\beta+1)}\left(t_{1}^{\alpha+\beta}+\frac{t_{1}^{\alpha+1}-t_{1}^{\alpha} \eta_{1}}{\zeta_{1}^{\alpha+1}-\zeta_{1}^{\alpha} \eta_{1}} \zeta_{1}^{\alpha+\beta}+\frac{t_{1}^{\alpha} \zeta_{1}-t_{1}^{\alpha+1}}{\zeta_{1} \eta_{1}^{\alpha}-\eta_{1}^{\alpha+1}} \eta_{1}^{\alpha+\beta}\right), \quad t \in\left[0, t_{1}\right], \\
\mid y(t) & -g_{i}\left(t, x\left(t_{i}^{-}\right)\right) \mid \leq \varepsilon, \quad t \in\left(t_{i}, s_{i}\right], i=1,2, \ldots, m, \\
\mid y(t) & -F_{i}(t)+c_{i 1}^{*} t^{\alpha+1}+c_{i 2}^{*} t^{\alpha}+c_{i 3}^{*} \mid \\
\leq & \frac{\varepsilon}{\Gamma(\alpha+\beta+1)}\left\{t_{i+1}^{\alpha+\beta}+\left|\mathcal{M}_{i}\right| t_{i+1}^{\alpha+1}\right. \\
& \times\left[\left(\zeta_{i+1}^{\alpha}-s_{i}^{\alpha}\right) \eta_{i+1}^{\alpha+\beta}+\left(\eta_{i+1}^{\alpha}-s_{i}^{\alpha}\right) \zeta_{i+1}^{\alpha+\beta}\right]+\left|\mathcal{M}_{i}\right| t_{i+1}^{\alpha}\left[\left(\eta_{i+1}^{\alpha+1}-s_{i}^{\alpha+1}\right) \zeta_{i+1}^{\alpha+\beta}\right. \\
& \left.+\left(\zeta_{i+1}^{\alpha+1}-s_{i}^{\alpha+1}\right) \eta_{i+1}^{\alpha+\beta}\right]+\left|\mathcal{M}_{i}\right|\left[\left(\zeta_{i+1}^{\alpha+1} s_{i}^{\alpha}-s_{i}^{\alpha+1} \zeta_{i+1}^{\alpha}\right) \eta_{i+1}^{\alpha+\beta}\right. \\
& \left.\left.+\left(\eta_{i+1}^{\alpha+1} s_{i}^{\alpha}-s_{i}^{\alpha+1} \eta_{i+1}^{\alpha}\right) \zeta_{i+1}^{\alpha+\beta}\right]\right\}, \quad t \in\left(s_{i}, t_{i+1}\right], i=1,2, \ldots, m .
\end{aligned}\right.
$$

Proof According to Remark 2.1, we have

$$
\left\{\begin{array}{l}
{ }^{c} D_{s_{i}^{+}}^{\beta}\left({ }^{c} D_{s_{i}^{+}}^{\alpha}+\mathcal{A}\right) y(t)=f\left(t, y(t),{ }^{c} D_{s_{i}^{+}}^{\alpha} y(t)\right)+\mathcal{B}(t), \quad t \in\left(s_{i}, t_{i+1}\right], i=0,1, \ldots, m, \\
y(t)=g_{i}\left(t, y\left(t_{i}^{-}\right)\right)+\mathcal{B}_{i}, \quad t \in\left(t_{i}, s_{i}\right], i=1,2, \ldots, m, \\
y(0)=y(T)=y\left(\zeta_{i+1}\right)=y\left(\eta_{i+1}\right)=0, \quad \eta_{i+1}, \zeta_{i+1} \in\left(s_{i}, t_{i+1}\right), i=0,1, \ldots, m .
\end{array}\right.
$$

By Lemma 2.2 and (2.12), we obtain

$$
y(t)= \begin{cases}\widetilde{F}_{0}(t)-\frac{t^{\alpha+1}-t^{\alpha} \eta_{1}}{\zeta_{1}^{\alpha+1}-\zeta_{1}^{\alpha} \eta_{1}} \widetilde{F}_{0}\left(\zeta_{1}\right)-\frac{t^{\alpha} \zeta_{1}-t^{\alpha+1}}{\zeta_{1} \eta_{1}^{\alpha}-\eta_{1}^{\alpha+1}} \widetilde{F}_{0}\left(\eta_{1}\right), & t \in\left[0, t_{1}\right], \\ g_{i}\left(t, y\left(s_{i}^{-}\right)\right)+\mathcal{B}_{i}, & t \in\left(t_{i}, s_{i}\right], i=1,2, \ldots, m, \\ \widetilde{F}_{i}(t)-\widetilde{c}_{i 1}^{*} t^{\alpha+1}-\widetilde{c}_{i 2}^{*} t^{\alpha}-\widetilde{c}_{i 3}^{*}, & t \in\left(s_{i}, t_{i+1}\right], i=1,2, \ldots, m,\end{cases}
$$

where

$$
\begin{aligned}
\widetilde{F}_{i}(t)= & \int_{s_{i}}^{t} \frac{(t-u)^{\alpha-1}}{\Gamma(\alpha)}\left(\int_{s_{i}}^{u} \frac{(u-s)^{\beta-1}}{\Gamma(\beta)}\left(f\left(s, y(s),{ }^{c} D_{s_{i}^{+}}^{\alpha} y(s)\right)+\mathcal{B}(s)\right) d s-(\mathcal{A} y)(u)\right) d u, \\
\widetilde{c}_{i 1}^{*}= & \mathcal{M}_{i}\left[\left(\zeta_{i+1}^{\alpha}-s_{i}^{\alpha}\right) \widetilde{F}_{i}\left(\eta_{i+1}\right)-\left(\eta_{i+1}^{\alpha}-s_{i}^{\alpha}\right) \widetilde{F}_{i}\left(\zeta_{i+1}\right)-\left(\zeta_{i+1}^{\alpha}-\eta_{i+1}^{\alpha}\right) g_{i}\left(s_{i}, y\left(t_{i}^{-}\right)\right)\right], \\
\widetilde{c}_{i 2}^{*}= & \mathcal{M}_{i}\left[\left(\eta_{i+1}^{\alpha+1}-s_{i}^{\alpha+1}\right) \widetilde{F}_{i}\left(\zeta_{i+1}\right)-\left(\zeta_{i+1}^{\alpha+1}-s_{i}^{\alpha+1}\right) \widetilde{F}_{i}\left(\eta_{i+1}\right)+\left(\eta_{i+1}^{\alpha+1}-\zeta_{i+1}^{\alpha+1}\right) g_{i}\left(s_{i}, y\left(t_{i}^{-}\right)\right)\right], \\
\widetilde{c}_{i 3}^{*}= & \mathcal{M}_{i}\left[\left(\zeta_{i+1}^{\alpha+1} s_{i}^{\alpha}-s_{i}^{\alpha+1} \zeta_{i+1}^{\alpha}\right) \widetilde{F}_{i}\left(\eta_{i+1}\right)-\left(\eta_{i+1}^{\alpha+1} s_{i}^{\alpha}-s_{i}^{\alpha+1} \eta_{i+1}^{\alpha}\right) \widetilde{F}_{i}\left(\zeta_{i+1}\right)\right. \\
& \left.-\left(\zeta_{i+1}^{\alpha+1} \eta_{i+1}^{\alpha}-\eta_{i+1}^{\alpha+1} \zeta_{i+1}^{\alpha}\right) g_{i}\left(s_{i}, y\left(t_{i}^{-}\right)\right)\right] .
\end{aligned}
$$

It is worth noticing that the Beta function $B(\cdot, \cdot)$ will be extensively used in the following calculation. So we introduce it as follows:

$$
\begin{aligned}
& B(\beta+1, \alpha)=\int_{0}^{1}(1-u)^{\alpha-1} u^{\beta} d u=\frac{\Gamma(\alpha) \Gamma(\beta+1)}{\Gamma(\alpha+\beta+1)} \\
& \int_{0}^{t}(t-u)^{\alpha-1} \int_{0}^{u}(u-s)^{\beta-1} d s d u=\int_{0}^{t}(t-u)^{\alpha-1} u^{\beta} d u=\frac{\Gamma(\alpha) \Gamma(\beta+1)}{\Gamma(\alpha+\beta+1)} t^{\alpha+\beta} .
\end{aligned}
$$


When $t \in\left[0, t_{1}\right]$, we have

$$
\begin{aligned}
& \left|y(t)-F_{0}(t)+\frac{t^{\alpha+1}-t^{\alpha} \eta_{1}}{\zeta_{1}^{\alpha+1}-\zeta_{1}^{\alpha} \eta_{1}} F_{0}\left(t_{1}\right)+\frac{t^{\alpha} \zeta_{1}-t^{\alpha+1}}{\zeta_{1} \eta_{1}^{\alpha}-\eta_{1}^{\alpha+1}} F_{0}\left(\eta_{1}\right)\right| \\
& =\mid \int_{0}^{t} \frac{(t-u)^{\alpha-1}}{\Gamma(\alpha)} \int_{0}^{u} \frac{(u-s)^{\beta-1}}{\Gamma(\beta)} \mathcal{B}(s) d s d u \\
& -\frac{t^{\alpha+1}-t^{\alpha} \eta_{1}}{\zeta_{1}^{\alpha+1}-\zeta_{1}^{\alpha} \eta_{1}} \int_{0}^{\zeta_{1}} \frac{\left(\zeta_{1}-u\right)^{\alpha-1}}{\Gamma(\alpha)} \int_{0}^{u} \frac{(u-s)^{\beta-1}}{\Gamma(\beta)} \mathcal{B}(s) d s d u \\
& -\frac{t^{\alpha} \zeta_{1}-t^{\alpha+1}}{\zeta_{1} \eta_{1}^{\alpha}-\eta_{1}^{\alpha+1}} \int_{0}^{\eta_{1}} \frac{\left(\eta_{1}-u\right)^{\alpha-1}}{\Gamma(\alpha)} \int_{0}^{u} \frac{(u-s)^{\beta-1}}{\Gamma(\beta)} \mathcal{B}(s) d s d u \\
& \leq \int_{0}^{t} \frac{(t-u)^{\alpha-1}}{\Gamma(\alpha)} \int_{0}^{u} \frac{(u-s)^{\beta-1}}{\Gamma(\beta)}|\mathcal{B}(s)| d s d u \\
& +\left|\frac{t^{\alpha+1}-t^{\alpha} \eta_{1}}{\zeta_{1}^{\alpha+1}-\zeta_{1}^{\alpha} \eta_{1}}\right| \int_{0}^{\zeta_{1}} \frac{\left(\zeta_{1}-u\right)^{\alpha-1}}{\Gamma(\alpha)} \int_{0}^{u} \frac{(u-s)^{\beta-1}}{\Gamma(\beta)}|\mathcal{B}(s)| d s d u \\
& +\left|\frac{t^{\alpha} \zeta_{1}-t^{\alpha+1}}{\zeta_{1} \eta_{1}^{\alpha}-\eta_{1}^{\alpha+1}}\right| \int_{0}^{\eta_{1}} \frac{\left(\eta_{1}-u\right)^{\alpha-1}}{\Gamma(\alpha)} \int_{0}^{u} \frac{(u-s)^{\beta-1}}{\Gamma(\beta)}|\mathcal{B}(s)| d s d u \\
& \leq \frac{\varepsilon t^{\alpha+\beta}}{\Gamma(\alpha+\beta+1)}+\frac{\varepsilon \zeta_{1}^{\alpha+\beta}}{\Gamma(\alpha+\beta+1)}\left|\frac{t^{\alpha+1}-t^{\alpha} \eta_{1}}{\zeta_{1}^{\alpha+1}-\zeta_{1}^{\alpha} \eta_{1}}\right|+\frac{\varepsilon \eta_{1}^{\alpha+\beta}}{\Gamma(\alpha+\beta+1)}+\left|\frac{t^{\alpha} \zeta_{1}-t^{\alpha+1}}{\zeta_{1} \eta_{1}^{\alpha}-\eta_{1}^{\alpha+1}}\right| \\
& \leq \frac{\varepsilon}{\Gamma(\alpha+\beta+1)}\left(t_{1}^{\alpha+\beta}+\frac{t_{1}^{\alpha+1}-t_{1}^{\alpha} \eta_{1}}{\zeta_{1}^{\alpha+1}-\zeta_{1}^{\alpha} \eta_{1}} \zeta_{1}^{\alpha+\beta}+\frac{t_{1}^{\alpha} \zeta_{1}-t_{1}^{\alpha+1}}{\zeta_{1} \eta_{1}^{\alpha}-\eta_{1}^{\alpha+1}} \eta_{1}^{\alpha+\beta}\right)
\end{aligned}
$$

When $t \in\left(t_{i}, s_{i}\right], i=1,2, \ldots, m$, we get

$$
\left|y(t)-g_{i}\left(t, y\left(t_{i}^{-}\right)\right)\right|=\left|\mathcal{B}_{i}\right| \leq \varepsilon .
$$

When $t \in\left(s_{i}, t_{i+1}\right], i=1,2, \ldots, m$, it follows that

$$
\begin{aligned}
\mid y(t) & -F_{i}(t)+c_{i 1}^{*} t^{\alpha+1}+c_{i 2}^{*} t^{\alpha}+c_{i 3}^{*} \mid \\
= & \mid \int_{s_{i}}^{t} \frac{(t-u)^{\alpha-1}}{\Gamma(\alpha)} \int_{s_{i}}^{u} \frac{(u-s)^{\beta-1}}{\Gamma(\beta)} \mathcal{B}(s) d s d u-\mathcal{M}_{i} t^{\alpha+1}\left[\left(\zeta_{i+1}^{\alpha}-s_{i}^{\alpha}\right)\left(\widetilde{F}_{i}\left(\eta_{i+1}\right)-F_{i}\left(\eta_{i+1}\right)\right)\right. \\
& \left.-\left(\eta_{i+1}^{\alpha}-s_{i}^{\alpha}\right)\left(\widetilde{F}_{i}\left(\zeta_{i+1}\right)-F_{i}\left(\zeta_{i+1}\right)\right)\right]-\mathcal{M}_{i} t^{\alpha}\left[\left(\eta_{i+1}^{\alpha+1}-s_{i}^{\alpha+1}\right)\left(\widetilde{F}_{i}\left(\zeta_{i+1}\right)-F_{i}\left(\zeta_{i+1}\right)\right)\right. \\
& \left.-\left(\zeta_{i+1}^{\alpha+1}-s_{i}^{\alpha+1}\right)\left(\widetilde{F}_{i}\left(\eta_{i+1}\right)-F_{i}\left(\eta_{i+1}\right)\right)\right]-\mathcal{M}_{i}\left[\left(\zeta_{i+1}^{\alpha+1} s_{i}^{\alpha}-s_{i}^{\alpha+1} \zeta_{i+1}^{\alpha}\right)\left(\widetilde{F}_{i}\left(\eta_{i+1}\right)-F_{i}\left(\eta_{i+1}\right)\right)\right. \\
& \left.-\left(\eta_{i+1}^{\alpha+1} s_{i}^{\alpha}-s_{i}^{\alpha+1} \eta_{i+1}^{\alpha}\right)\left(\widetilde{F}_{i}\left(\zeta_{i+1}\right)-F_{i}\left(\zeta_{i+1}\right)\right)\right] \mid \\
\leq & \int_{s_{i}}^{t} \frac{(t-u)^{\alpha-1}}{\Gamma(\alpha)} \int_{s_{i}}^{u} \frac{(u-s)^{\beta-1}}{\Gamma(\beta)}|\mathcal{B}(s)| d s d u \\
& +\left|\mathcal{M}_{i}\right| t^{\alpha+1}\left[\left(\zeta_{i+1}^{\alpha}-s_{i}^{\alpha}\right) \int_{s_{i}}^{\eta_{i+1}} \frac{\left(\eta_{i+1}-u\right)^{\alpha-1}}{\Gamma(\alpha)} \int_{s_{i}}^{u} \frac{(u-s)^{\beta-1}}{\Gamma(\beta)}|\mathcal{B}(s)| d s d u\right. \\
& \left.+\left(\eta_{i+1}^{\alpha}-s_{i}^{\alpha}\right) \int_{s_{i}}^{\zeta_{i+1}} \frac{\left(\zeta_{i+1}-u\right)^{\alpha-1}}{\Gamma(\alpha)} \int_{s_{i}}^{u} \frac{(u-s)^{\beta-1}}{\Gamma(\beta)}|\mathcal{B}(s)| d s d u\right] \\
& +\left|\mathcal{M}_{i}\right| t^{\alpha}\left[\left(\eta_{i+1}^{\alpha+1}-s_{i}^{\alpha+1}\right) \int_{s_{i}}^{\zeta_{i+1}} \frac{\left(\zeta_{i+1}-u\right)^{\alpha-1}}{\Gamma(\alpha)} \int_{s_{i}}^{u} \frac{(u-s)^{\beta-1}}{\Gamma(\beta)}|\mathcal{B}(s)| d s d u\right.
\end{aligned}
$$




$$
\begin{aligned}
& \left.+\left(\zeta_{i+1}^{\alpha+1}-s_{i}^{\alpha+1}\right) \int_{s_{i}}^{\eta_{i+1}} \frac{\left(\eta_{i+1}-u\right)^{\alpha-1}}{\Gamma(\alpha)} \int_{s_{i}}^{u} \frac{(u-s)^{\beta-1}}{\Gamma(\beta)}|\mathcal{B}(s)| d s d u\right] \\
& +\left|\mathcal{M}_{i}\right|\left[\left(\zeta_{i+1}^{\alpha+1} s_{i}^{\alpha}-s_{i}^{\alpha+1} \zeta_{i+1}^{\alpha}\right) \int_{s_{i}}^{\eta_{i+1}} \frac{\left(\eta_{i+1}-u\right)^{\alpha-1}}{\Gamma(\alpha)} \int_{s_{i}}^{u} \frac{(u-s)^{\beta-1}}{\Gamma(\beta)}|\mathcal{B}(s)| d s d u\right. \\
& \left.+\left(\eta_{i+1}^{\alpha+1} s_{i}^{\alpha}-s_{i}^{\alpha+1} \eta_{i+1}^{\alpha}\right) \int_{s_{i}}^{\zeta_{i+1}} \frac{\left(\zeta_{i+1}-u\right)^{\alpha-1}}{\Gamma(\alpha)} \int_{s_{i}}^{u} \frac{(u-s)^{\beta-1}}{\Gamma(\beta)}|\mathcal{B}(s)| d s d u\right] \\
& \leq \varepsilon \int_{0}^{t} \frac{(t-u)^{\alpha-1}}{\Gamma(\alpha)} \int_{0}^{u} \frac{(u-s)^{\beta-1}}{\Gamma(\beta)} d s d u \\
& +\varepsilon\left|\mathcal{M}_{i}\right| t^{\alpha+1}\left[\left(\zeta_{i+1}^{\alpha}-s_{i}^{\alpha}\right) \int_{0}^{\eta_{i+1}} \frac{\left(\eta_{i+1}-u\right)^{\alpha-1}}{\Gamma(\alpha)} \int_{0}^{u} \frac{(u-s)^{\beta-1}}{\Gamma(\beta)} d s d u\right. \\
& \left.+\left(\eta_{i+1}^{\alpha}-s_{i}^{\alpha}\right) \int_{0}^{\zeta_{i+1}} \frac{\left(\zeta_{i+1}-u\right)^{\alpha-1}}{\Gamma(\alpha)} \int_{0}^{u} \frac{(u-s)^{\beta-1}}{\Gamma(\beta)} d s d u\right] \\
& +\varepsilon\left|\mathcal{M}_{i}\right| t^{\alpha}\left[\left(\eta_{i+1}^{\alpha+1}-s_{i}^{\alpha+1}\right) \int_{0}^{\zeta_{i+1}} \frac{\left(\zeta_{i+1}-u\right)^{\alpha-1}}{\Gamma(\alpha)} \int_{0}^{u} \frac{(u-s)^{\beta-1}}{\Gamma(\beta)} d s d u\right. \\
& \left.+\left(\zeta_{i+1}^{\alpha+1}-s_{i}^{\alpha+1}\right) \int_{0}^{\eta_{i+1}} \frac{\left(\eta_{i+1}-u\right)^{\alpha-1}}{\Gamma(\alpha)} \int_{0}^{u} \frac{(u-s)^{\beta-1}}{\Gamma(\beta)} d s d u\right] \\
& +\varepsilon\left|\mathcal{M}_{i}\right|\left[\left(\zeta_{i+1}^{\alpha+1} s_{i}^{\alpha}-s_{i}^{\alpha+1} \zeta_{i+1}^{\alpha}\right) \int_{0}^{\eta_{i+1}} \frac{\left(\eta_{i+1}-u\right)^{\alpha-1}}{\Gamma(\alpha)} \int_{0}^{u} \frac{(u-s)^{\beta-1}}{\Gamma(\beta)} d s d u\right. \\
& \left.+\left(\eta_{i+1}^{\alpha+1} s_{i}^{\alpha}-s_{i}^{\alpha+1} \eta_{i+1}^{\alpha}\right) \int_{0}^{\zeta_{i+1}} \frac{\left(\zeta_{i+1}-u\right)^{\alpha-1}}{\Gamma(\alpha)} \int_{0}^{u} \frac{(u-s)^{\beta-1}}{\Gamma(\beta)} d s d u\right] \\
& =\frac{\varepsilon t^{\alpha+\beta}}{\Gamma(\alpha+\beta+1)}+\frac{\varepsilon\left|\mathcal{M}_{i}\right| t^{\alpha+1}}{\Gamma(\alpha+\beta+1)}\left[\left(\zeta_{i+1}^{\alpha}-s_{i}^{\alpha}\right) \eta_{i+1}^{\alpha+\beta}+\left(\eta_{i+1}^{\alpha}-s_{i}^{\alpha}\right) \zeta_{i+1}^{\alpha+\beta}\right] \\
& +\frac{\varepsilon\left|\mathcal{M}_{i}\right| t^{\alpha}}{\Gamma(\alpha+\beta+1)}\left[\left(\eta_{i+1}^{\alpha+1}-s_{i}^{\alpha+1}\right) \zeta_{i+1}^{\alpha+\beta}+\left(\zeta_{i+1}^{\alpha+1}-s_{i}^{\alpha+1}\right) \eta_{i+1}^{\alpha+\beta}\right] \\
& +\frac{\varepsilon\left|\mathcal{M}_{i}\right|}{\Gamma(\alpha+\beta+1)}\left[\left(\zeta_{i+1}^{\alpha+1} s_{i}^{\alpha}-s_{i}^{\alpha+1} \zeta_{i+1}^{\alpha}\right) \eta_{i+1}^{\alpha+\beta}+\left(\eta_{i+1}^{\alpha+1} s_{i}^{\alpha}-s_{i}^{\alpha+1} \eta_{i+1}^{\alpha}\right) \zeta_{i+1}^{\alpha+\beta}\right] \\
& \leq \frac{\varepsilon}{\Gamma(\alpha+\beta+1)}\left\{t_{i+1}^{\alpha+\beta}+\left|\mathcal{M}_{i}\right| t_{i+1}^{\alpha+1}\left[\left(\zeta_{i+1}^{\alpha}-s_{i}^{\alpha}\right) \eta_{i+1}^{\alpha+\beta}+\left(\eta_{i+1}^{\alpha}-s_{i}^{\alpha}\right) \zeta_{i+1}^{\alpha+\beta}\right]\right. \\
& +\left|\mathcal{M}_{i}\right| t_{i+1}^{\alpha}\left[\left(\eta_{i+1}^{\alpha+1}-s_{i}^{\alpha+1}\right) \zeta_{i+1}^{\alpha+\beta}+\left(\zeta_{i+1}^{\alpha+1}-s_{i}^{\alpha+1}\right) \eta_{i+1}^{\alpha+\beta}\right] \\
& \left.+\left|\mathcal{M}_{i}\right|\left[\left(\zeta_{i+1}^{\alpha+1} s_{i}^{\alpha}-s_{i}^{\alpha+1} \zeta_{i+1}^{\alpha}\right) \eta_{i+1}^{\alpha+\beta}+\left(\eta_{i+1}^{\alpha+1} s_{i}^{\alpha}-s_{i}^{\alpha+1} \eta_{i+1}^{\alpha}\right) \zeta_{i+1}^{\alpha+\beta}\right]\right\} .
\end{aligned}
$$

Thus we conclude that the inequality (2.11) holds. The proof is completed.

\section{Existence and Ulam-Hyers stability}

In this section, we shall discuss the existence and Ulam-Hyers stability for the problem (1.1). Now we introduce some assumptions to ensure that the problem (1.1) has a unique solution which is Ulam-Hyers stable. So consider

$\left(H_{1}\right) f \in C^{1}\left(J \times \mathbb{R}^{2}, \mathbb{R}\right)$ and there exist some constants $L_{1 f}, L_{2 f}>0$ such that

$$
\begin{aligned}
& \left|f\left(t, \omega_{1}, \bar{\omega}_{1}\right)-f\left(t, \omega_{2}, \bar{\omega}_{2}\right)\right| \\
& \quad \leq L_{1 f}\left|\omega_{1}-\omega_{2}\right|+L_{2 f}\left|\bar{\omega}_{1}-\bar{\omega}_{2}\right|, \quad t \in J, \omega_{1}, \bar{\omega}_{1}, \omega_{2}, \bar{\omega}_{2} \in \mathbb{R} ;
\end{aligned}
$$


$\left(H_{2}\right) g_{i} \in C\left(\left(t_{i}, s_{i}\right] \times \mathbb{R}, \mathbb{R}\right)$ and there exist some constants $L_{g_{i}}>0, i=1,2, \ldots, m$ such that

$$
\left|g_{i}(t, \omega)-g_{i}(t, \bar{\omega})\right| \leq L_{g_{i}}|\omega-\bar{\omega}|, \quad t \in\left(t_{i}, s_{i}\right], \omega, \bar{\omega} \in \mathbb{R}
$$

$\left(H_{3}\right) \mathcal{A} \in C^{2}(\mathbb{R}, \mathbb{R})$ and there exists a constant $L_{\mathcal{A}}>0$ such that

$$
|\mathcal{A} x-\mathcal{A} y| \leq L_{\mathcal{A}}|x-y|, \quad t \in J, x, y \in \mathbb{R}
$$

$\left(H_{4}\right) 0<\lambda<1$, where $\lambda=\max \left\{\lambda_{0}, \lambda_{1}, \ldots, \lambda_{m}, \mu_{0}, \mu_{1}, \ldots, \mu_{m}, L_{g_{1}}, \ldots, L_{g_{m}}\right\}$,

$$
\begin{aligned}
\lambda_{0}= & \frac{L_{1 f}+L_{2 f}}{\Gamma(\alpha+\beta+1)}\left(t_{1}^{\alpha+\beta}+\frac{t_{1}^{\alpha+1}-t_{1}^{\alpha} \eta_{1}}{\zeta_{1}^{\alpha+1}-\zeta_{1}^{\alpha} \eta_{1}} \zeta_{1}^{\alpha+\beta}+\frac{t_{1}^{\alpha+1}-t_{1}^{\alpha} \zeta_{1}}{\left.\eta_{1}^{\alpha} \zeta_{1}-\eta_{1}^{\alpha+1} \eta_{1}^{\alpha+\beta}\right)}\right. \\
& +\frac{L_{\mathcal{A}}}{\Gamma(\alpha+1)}\left(t_{1}^{\alpha}+\frac{t_{1}^{\alpha+1}-t_{1}^{\alpha} \eta_{1}}{\zeta_{1}^{\alpha+1}-\zeta_{1}^{\alpha} \eta_{1}^{\alpha}} \zeta_{1}^{\alpha}+\frac{t_{1}^{\alpha+1}-t_{1}^{\alpha} \zeta_{1}}{\eta_{1}^{\alpha} \zeta_{1}-\eta_{1}^{\alpha+1}} \eta_{1}^{\alpha}\right), \\
\mu_{0}= & \left(L_{1 f}+L_{2 f}\right)\left[\frac{t_{1}^{\beta}}{\Gamma(\beta+1)}+\frac{1}{\Gamma(\alpha+\beta+1)}\left(\frac{t_{1} \Gamma(\alpha+2)-\Gamma(\alpha+1) \eta_{1}}{\zeta_{1}^{\alpha+1}-\zeta_{1}^{\alpha} \eta_{1}} \zeta_{1}^{\alpha+\beta}\right.\right. \\
& \left.\left.+\frac{t_{1} \Gamma(\alpha+2)-\Gamma(\alpha+1) \zeta_{1}}{\eta_{1}^{\alpha} \zeta_{1}-\eta_{1}^{\alpha+1}} \eta_{1}^{\alpha+\beta}\right)\right]+L_{\mathcal{A}}\left[1+\frac{1}{\Gamma(\alpha+1)}\right. \\
& \left.\times\left(\frac{t_{1} \Gamma(\alpha+2)-\Gamma(\alpha+1) \eta_{1}}{\zeta_{1}^{\alpha+1}-\zeta_{1}^{\alpha} \eta_{1}} \zeta_{1}^{\alpha}+\frac{t_{1} \Gamma(\alpha+2)-\Gamma(\alpha+1) \zeta_{1}}{\eta_{1}^{\alpha} \zeta_{1}-\eta_{1}^{\alpha+1}} \eta_{1}^{\alpha}\right)\right] \\
& \frac{L_{1 f}+L_{2 f}}{\Gamma(\alpha+\beta+1)}\left[t_{i+1}^{\alpha+\beta}+\left|\mathcal{M}_{i}\right| t_{i+1}^{\alpha+1}\left[\left(\zeta_{i+1}^{\alpha}-s_{i}^{\alpha}\right) \eta_{i+1}^{\alpha+\beta}+\left(\eta_{i+1}^{\alpha}-s_{i}^{\alpha}\right) \zeta_{i+1}^{\alpha+\beta}\right]\right. \\
& +\left|\mathcal{M}_{i}\right| t_{i+1}^{\alpha}\left[\left(\eta_{i+1}^{\alpha+1}-s_{i}^{\alpha+1}\right) \zeta_{i+1}^{\alpha+\beta}+\left(\zeta_{i+1}^{\alpha+1}-s_{i}^{\alpha+1}\right) \eta_{i+1}^{\alpha+\beta}\right]+\left|\mathcal{M}_{i}\right|\left[\left(\zeta_{i+1}^{\alpha+1} s_{i}^{\alpha}\right.\right. \\
& \left.\left.\left.-s_{i}^{\alpha+1} \zeta_{i+1}^{\alpha}\right) \eta_{i+1}^{\alpha+\beta}+\left(\eta_{i+1}^{\alpha+1} s_{i}^{\alpha}-s_{i}^{\alpha+1} \eta_{i+1}^{\alpha}\right) \zeta_{i+1}^{\alpha+\beta}\right]\right]+\frac{L_{\mathcal{A}}}{\Gamma(\alpha+1)}\left[t_{i+1}^{\alpha}\right. \\
& +\left|\mathcal{M}_{i}\right| t_{i+1}^{\alpha+1}\left[\left(\zeta_{i+1}^{\alpha}-s_{i}^{\alpha}\right) \eta_{i+1}^{\alpha}+\left(\eta_{i+1}^{\alpha}-s_{i}^{\alpha}\right) \zeta_{i+1}^{\alpha}\right]+\left|\mathcal{M}_{i}\right| t_{i+1}^{\alpha}\left[\left(\eta_{i+1}^{\alpha}-s_{i}^{\alpha}\right) \zeta_{i+1}^{\alpha}\right. \\
& \left.+\left(\zeta_{i+1}^{\alpha+1}-s_{i}^{\alpha+1}\right) \eta_{i+1}^{\alpha}\right]+\left|\mathcal{M}_{i}\right|\left[\left(\zeta_{i+1}^{\alpha+1} s_{i}^{\alpha}-s_{i}^{\alpha+1} \zeta_{i+1}^{\alpha}\right) \eta_{i+1}^{\alpha}+\left(\eta_{i+1}^{\alpha+1} s_{i}^{\alpha}\right.\right. \\
& \left.\left.\left.-s_{i}^{\alpha+1} \eta_{i+1}^{\alpha}\right) \zeta_{i+1}^{\alpha}\right]\right]+L_{g_{i}} \mathcal{M}_{i}\left[t_{i+1}^{\alpha+1}\left(\zeta_{i+1}^{\alpha}-\eta_{i+1}^{\alpha}\right)+t_{i+1}^{\alpha}\left(\zeta_{i+1}^{\alpha+1}-\eta_{i+1}^{\alpha+1}\right)\right. \\
& \left.+\left(\zeta_{i+1}^{\alpha+1} \eta_{i+1}^{\alpha}-\eta_{i+1}^{\alpha+1} \zeta_{i+1}^{\alpha}\right)\right], \\
\mu_{i}= & \left(L_{1 f}+L_{2 f}\right) \\
& +\left(\frac{t_{i+1}^{\beta}}{\Gamma(\beta+1)}+\frac{\left|\mathcal{M}_{i}\right| t_{i+1} \Gamma(\alpha+2)}{\Gamma(\alpha+\beta+1)}\left[\left(\zeta_{i+1}^{\alpha}-s_{i}^{\alpha}\right) \eta_{i+1}^{\alpha+\beta}+\left(\eta_{i+1}^{\alpha}-s_{i}^{\alpha}\right) \zeta_{i+1}^{\alpha+\beta}\right]\right. \\
& \left.+\frac{\left|\mathcal{M}_{i}\right|}{\Gamma(\alpha+\beta+1)}\left[\left(\zeta_{i+1}^{\alpha+1}-s_{i}^{\alpha+1}\right) \eta_{i+1}^{\alpha+\beta}+\left(\eta_{i+1}^{\alpha+1}-s_{i}^{\alpha+1}\right) \zeta_{i+1}^{\alpha+\beta}\right]\right) \\
& +L_{g_{i}}\left|\mathcal{M}_{i}\right|\left[t _ { i + 1 } \Gamma \left(\alpha+\left|\mathcal{M}_{i}\right| t_{i+1}(\alpha+1)\left[\left(\zeta_{i+1}^{\alpha}-s_{i}^{\alpha}\right) \eta_{i+1}^{\alpha}+\left(\eta_{i+1}^{\alpha}-s_{i}^{\alpha}\right) \zeta_{i+1}^{\alpha}\right]\right.\right. \\
& \\
&
\end{aligned}
$$

Theorem 3.1 Assume that $\left(H_{1}\right)-\left(H_{4}\right)$ hold. Then the following assertions are true: 
(1) The problem (1.1) has a unique solution $y_{0} \in X$ and satisfies the integral equation (2.1), namely,

$$
y_{0}(t)= \begin{cases}F_{0}(t)-\frac{t^{\alpha+1}-t^{\alpha} \eta_{1}}{\zeta_{1}^{\alpha+1}-\zeta_{1}^{\alpha} \eta_{1}} F_{0}\left(\zeta_{1}\right)-\frac{t^{\alpha} \zeta_{1}-t^{\alpha+1}}{\zeta_{1} \eta_{1}^{\alpha}-\eta_{1}^{\alpha+1}} F_{0}\left(\eta_{1}\right), & t \in\left[0, t_{1}\right], \\ g_{i}\left(t, y\left(s_{i}^{-}\right)\right), & t \in\left(t_{i}, s_{i}\right], i=1,2, \ldots, m, \\ F_{i}(t)-c_{i 1}^{*} t^{\alpha+1}-c_{i 2}^{*} t^{\alpha}-c_{i 3}^{*}, & t \in\left(s_{i}, t_{i+1}\right], i=1,2, \ldots, m,\end{cases}
$$

where $c_{i 1}^{*}, c_{i 2}^{*}$, and $c_{i 3}^{*}$ are the same as in (2.1), and

$$
F_{i}(t)=\int_{s_{i}}^{t} \frac{(t-u)^{\alpha-1}}{\Gamma(\alpha)}\left(\int_{s_{i}}^{u} \frac{(u-s)^{\beta-1}}{\Gamma(\beta)} f\left(s, y_{0}(s),{ }^{c} D_{s_{i}^{+}}^{\alpha} y_{0}(s)\right) d s-\left(\mathcal{A} y_{0}\right)(u)\right) d u .
$$

(2) The problem (1.1) is Ulam-Hyers stable, that is, if $y \in X$ is a solution of the inequality (2.10), then

$$
\left|y(t)-y_{0}(t)\right| \leq \kappa \varepsilon, \quad t \in J
$$

where $\kappa=\max \left\{1, \kappa_{0}, \kappa_{1}, \ldots, \kappa_{m}\right\}$,

$$
\begin{aligned}
\kappa_{0}= & \frac{1}{\Gamma(\alpha+\beta+1)}\left(t_{1}^{\alpha+\beta}+\frac{t_{1}^{\alpha+1}-t_{1}^{\alpha} \eta_{1}}{\zeta_{1}^{\alpha+1}-\zeta_{1}^{\alpha} \eta_{1}} \zeta_{1}^{\alpha+\beta}+\frac{t_{1}^{\alpha} \zeta_{1}-t_{1}^{\alpha+1}}{\zeta_{1} \eta_{1}^{\alpha}-\eta_{1}^{\alpha+1}} \eta_{1}^{\alpha+\beta}\right), \\
\kappa_{i}= & \frac{1}{\Gamma(\alpha+\beta+1)}\left\{t_{i+1}^{\alpha+\beta}+\left|\mathcal{M}_{i}\right| t_{i+1}^{\alpha+1}\left[\left(\zeta_{i+1}^{\alpha}-s_{i}^{\alpha}\right) \eta_{i+1}^{\alpha+\beta}+\left(\eta_{i+1}^{\alpha}-s_{i}^{\alpha}\right) \zeta_{i+1}^{\alpha+\beta}\right]\right. \\
& +\left|\mathcal{M}_{i}\right| t_{i+1}^{\alpha}\left[\left(\eta_{i+1}^{\alpha+1}-s_{i}^{\alpha+1}\right) \zeta_{i+1}^{\alpha+\beta}+\left(\zeta_{i+1}^{\alpha+1}-s_{i}^{\alpha+1}\right) \eta_{i+1}^{\alpha+\beta}\right] \\
& \left.+\left|\mathcal{M}_{i}\right|\left[\left(\zeta_{i+1}^{\alpha+1} s_{i}^{\alpha}-s_{i}^{\alpha+1} \zeta_{i+1}^{\alpha}\right) \eta_{i+1}^{\alpha+\beta}+\left(\eta_{i+1}^{\alpha+1} s_{i}^{\alpha}-s_{i}^{\alpha+1} \eta_{i+1}^{\alpha}\right) \zeta_{i+1}^{\alpha+\beta}\right]\right\} .
\end{aligned}
$$

Proof For all $x \in X, t \in J$, we define the operator $Q: X \rightarrow X$ by

$$
(Q x)(t)= \begin{cases}F_{0}(t)-\frac{t^{\alpha+1}-t^{\alpha} \eta_{1}}{\zeta_{1}^{\alpha+1}-\zeta_{1}^{\alpha} \eta_{1}} F_{0}\left(\zeta_{1}\right)-\frac{t^{\alpha} \zeta_{1}-t^{\alpha+1}}{\zeta_{1} \eta_{1}^{\alpha}-\eta_{1}^{\alpha+1}} F_{0}\left(\eta_{1}\right), & t \in\left[0, t_{1}\right], \\ g_{i}\left(t, x\left(s_{i}^{-}\right)\right), & t \in\left(t_{i}, s_{i}\right], i=1,2, \ldots, m, \\ F_{i}(t)-c_{i 1}^{*} t^{\alpha+1}-c_{i 2}^{*} t^{\alpha}-c_{i 3}^{*}, & t \in\left(s_{i}, t_{i+1}\right], i=1,2, \ldots, m .\end{cases}
$$

Since $x(t) \in X, f \in C^{1}\left(J \times \mathbb{R}^{2}, \mathbb{R}\right), g_{i} \in C\left(\left(t_{i}, s_{i}\right] \times \mathbb{R}, \mathbb{R}\right), \mathcal{A} \in C^{2}(\mathbb{R}, \mathbb{R})$, and due to the expression of $(Q x)(t)$, we know that if $x(t) \in X$, then $(Q x)(t) \in X$.

We firstly apply the Banach contraction principle to prove that (1) of Theorem 3.1 holds. To do so, we need to prove that $Q$ is strictly contractive on $X$. In fact, for any $v_{1}, v_{2} \in X$, in view of $\left(H_{1}\right)-\left(H_{3}\right)$, we have

$$
\begin{aligned}
& \left|\left(Q v_{1}\right)(t)-\left(Q v_{2}\right)(t)\right| \\
& \leq \int_{0}^{t} \frac{(t-u)^{\alpha-1}}{\Gamma(\alpha)}\left(\int_{0}^{u} \frac{(u-s)^{\beta-1}}{\Gamma(\beta)} \mid f\left(s, v_{1}(s),{ }^{c} D_{0^{+}}^{\alpha} v_{1}(s)\right)\right. \\
& \left.\quad-f\left(s, v_{2}(s),{ }^{c} D_{0^{+}}^{\alpha} v_{2}(s)\right)|d s+|\left(\mathcal{A} v_{1}\right)(u)-\left(\mathcal{A} v_{2}\right)(u) \mid\right) d u
\end{aligned}
$$




$$
\begin{aligned}
& +\left|\frac{t^{\alpha+1}-t^{\alpha} \eta_{1}}{\zeta_{1}^{\alpha+1}-\zeta_{1}^{\alpha} \eta_{1}}\right| \int_{0}^{\zeta_{1}} \frac{\left(\zeta_{1}-u\right)^{\alpha-1}}{\Gamma(\alpha)}\left(\int_{0}^{u} \frac{(u-s)^{\beta-1}}{\Gamma(\beta)} \mid f\left(s, v_{1}(s),{ }^{c} D_{0^{+}}^{\alpha} v_{1}(s)\right)\right. \\
& \left.-f\left(s, v_{2}(s),{ }^{c} D_{0^{+}}^{\alpha} v_{2}(s)\right)|d s+|\left(\mathcal{A} v_{1}\right)(u)-\left(\mathcal{A} v_{2}\right)(u) \mid\right) d u \\
& +\left|\frac{t^{\alpha} \zeta_{1}-t^{\alpha+1}}{\zeta_{1} \eta_{1}^{\alpha}-\eta_{1}^{\alpha+1}}\right| \int_{0}^{\eta_{1}} \frac{\left(\eta_{1}-u\right)^{\alpha-1}}{\Gamma(\alpha)}\left(\int_{0}^{u} \frac{(u-s)^{\beta-1}}{\Gamma(\beta)} \mid f\left(s, v_{1}(t),{ }^{c} D_{0^{+}}^{\alpha} v_{1}(s)\right)\right. \\
& \left.-f\left(s, v_{2}(s),{ }^{c} D_{0^{+}}^{\alpha} v_{2}(s)\right)|d s+|\left(\mathcal{A} v_{1}\right)(u)-\left(\mathcal{A} v_{2}\right)(u) \mid\right) d u \\
& \leq \int_{0}^{t} \frac{(t-u)^{\alpha-1}}{\Gamma(\alpha)}\left(\int _ { 0 } ^ { u } \frac { ( u - s ) ^ { \beta - 1 } } { \Gamma ( \beta ) } \left[L_{1 f}\left|v_{1}(s)-v_{2}(s)\right|\right.\right. \\
& \left.\left.\left.\left.+L_{2 f} \mid{ }^{c} D_{0^{+}}^{\alpha} v_{1}(s)\right)-{ }^{c} D_{0^{+}}^{\alpha} v_{2}(s)\right) \mid\right] d s+L_{\mathcal{A}}\left|v_{1}(u)-v_{2}(u)\right|\right) d u \\
& +\left|\frac{t^{\alpha+1}-t^{\alpha} \eta_{1}}{\zeta_{1}^{\alpha+1}-\zeta_{1}^{\alpha} \eta_{1}}\right| \int_{0}^{\zeta_{1}} \frac{\left(\zeta_{1}-u\right)^{\alpha-1}}{\Gamma(\alpha)}\left(\int _ { 0 } ^ { u } \frac { ( u - s ) ^ { \beta - 1 } } { \Gamma ( \beta ) } \left[L_{1 f}\left|v_{1}(s)-v_{2}(s)\right|\right.\right. \\
& \left.\left.\left.\left.+L_{2 f} \mid{ }^{c} D_{0^{+}}^{\alpha} v_{1}(s)\right)-{ }^{c} D_{0^{+}}^{\alpha} \nu_{2}(s)\right) \mid\right] d s+L_{\mathcal{A}}\left|v_{1}(u)-v_{2}(u)\right|\right) d u \\
& +\left|\frac{t^{\alpha} \zeta_{1}-t^{\alpha+1}}{\zeta_{1} \eta_{1}^{\alpha}-\eta_{1}^{\alpha+1}}\right| \int_{0}^{\eta_{1}} \frac{\left(\eta_{1}-u\right)^{\alpha-1}}{\Gamma(\alpha)}\left(\int _ { 0 } ^ { u } \frac { ( u - s ) ^ { \beta - 1 } } { \Gamma ( \beta ) } \left[L_{1 f}\left|v_{1}(s)-v_{2}(s)\right|\right.\right. \\
& \left.\left.\left.\left.+L_{2 f} \mid{ }^{c} D_{0^{+}}^{\alpha} v_{1}(s)\right)-{ }^{c} D_{0^{+}}^{\alpha} v_{2}(s)\right) \mid\right] d s+L_{\mathcal{A}}\left|v_{1}(u)-v_{2}(u)\right|\right) d u \\
& \leq\left(\frac{\left(L_{1 f}+L_{2 f}\right) t^{\alpha+\beta}}{\Gamma(\alpha+\beta+1)}+\frac{L_{\mathcal{A}} t^{\alpha}}{\Gamma(\alpha+1)}\right)\left\|v_{1}-v_{2}\right\|_{X}+\left(\frac{\left(L_{1 f}+L_{2 f}\right) \zeta_{1}^{\alpha+\beta}}{\Gamma(\alpha+\beta+1)}\right. \\
& \left.+\frac{L_{\mathcal{A}} \zeta_{1}^{\alpha}}{\Gamma(\alpha+1)}\right) \frac{t_{1}^{\alpha+1}-t_{1}^{\alpha} \eta_{1}}{\zeta_{1}^{\alpha+1}-\zeta_{1}^{\alpha} \eta_{1}}\left\|v_{1}-v_{2}\right\|_{X}+\left(\frac{\left(L_{1 f}+L_{2 f}\right) \eta_{1}^{\alpha+\beta}}{\Gamma(\alpha+\beta+1)}\right. \\
& \left.+\frac{L_{\mathcal{A}} \eta_{1}^{\alpha}}{\Gamma(\alpha+1)}\right) \frac{t_{1}^{\alpha+1}-t_{1}^{\alpha} \zeta_{1}}{\eta_{1}^{\alpha} \zeta_{1}-\eta_{1}^{\alpha+1}}\left\|v_{1}-v_{2}\right\|_{X} \\
& \leq\left\{\frac{L_{1 f}+L_{2 f}}{\Gamma(\alpha+\beta+1)}\left(t_{1}^{\alpha+\beta}+\frac{t_{1}^{\alpha+1}-t_{1}^{\alpha} \eta_{1}}{\zeta_{1}^{\alpha+1}-\zeta_{1}^{\alpha} \eta_{1}} \zeta_{1}^{\alpha+\beta}+\frac{t_{1}^{\alpha+1}-t_{1}^{\alpha} \zeta_{1}}{\eta_{1}^{\alpha} \zeta_{1}-\eta_{1}^{\alpha+1}} \eta_{1}^{\alpha+\beta}\right)\right. \\
& \left.+\frac{L_{\mathcal{A}}}{\Gamma(\alpha+1)}\left(t_{1}^{\alpha}+\frac{t_{1}^{\alpha+1}-t_{1}^{\alpha} \eta_{1}}{\zeta_{1}^{\alpha+1}-\zeta_{1}^{\alpha} \eta_{1}} \zeta_{1}^{\alpha}+\frac{t_{1}^{\alpha+1}-t_{1}^{\alpha} \zeta_{1}}{\eta_{1}^{\alpha} \zeta_{1}-\eta_{1}^{\alpha+1}} \eta_{1}^{\alpha}\right)\right\}\left\|v_{1}-v_{2}\right\|_{X} \\
& =\lambda_{0}\left\|v_{1}-v_{2}\right\|_{X}, \quad t \in\left[0, t_{1}\right] \\
& \left|{ }^{c} D_{0^{+}}^{\alpha}\left(Q v_{1}\right)(t)-{ }^{c} D_{0^{+}}^{\alpha}\left(Q v_{2}\right)(t)\right| \\
& \leq \int_{0}^{t} \frac{(t-s)^{\beta-1}}{\Gamma(\beta)}\left|f\left(s, v_{1}(s),{ }^{c} D_{0^{+}}^{\alpha} v_{1}(s)\right)-f\left(s, v_{2}(s),{ }^{c} D_{0^{+}}^{\alpha} v_{2}(s)\right)\right| d s \\
& +\left|\left(\mathcal{A} v_{1}\right)(t)-\left(\mathcal{A} v_{2}\right)(t)\right|+\left|\frac{1}{\Gamma(1-\alpha)} \int_{0}^{t}(t-s)^{-\alpha}\left(\frac{s^{\alpha+1}-s^{\alpha} \eta_{1}}{\zeta_{1}^{\alpha+1}-\zeta_{1}^{\alpha} \eta_{1}}\right)^{\prime} d s\right| \\
& \times \int_{0}^{\zeta_{1}} \frac{\left(\zeta_{1}-u\right)^{\alpha-1}}{\Gamma(\alpha)}\left(\int_{0}^{u} \frac{(u-s)^{\beta-1}}{\Gamma(\beta)} \mid f\left(s, v_{1}(s),{ }^{c} D_{0^{+}}^{\alpha} v_{1}(s)\right)\right. \\
& \left.-f\left(s, v_{2}(s),{ }^{c} D_{0^{+}}^{\alpha} v_{2}(s)\right)|d s+|\left(\mathcal{A} v_{1}\right)(u)-\left(\mathcal{A} v_{2}\right)(u) \mid\right) d u
\end{aligned}
$$




$$
\begin{aligned}
& +\left|\frac{1}{\Gamma(1-\alpha)} \int_{0}^{t}(t-s)^{-\alpha}\left(\frac{s^{\alpha} \zeta_{1}-s^{\alpha+1}}{\zeta_{1} \eta_{1}^{\alpha}-\eta_{1}^{\alpha+1}}\right)^{\prime} d s\right| \int_{0}^{\eta_{1}} \frac{\left(\eta_{1}-u\right)^{\alpha-1}}{\Gamma(\alpha)} \\
& \times\left(\int_{0}^{u} \frac{(u-s)^{\beta-1}}{\Gamma(\beta)}\left|f\left(s, v_{1}(t),{ }^{c} D_{0^{+}}^{\alpha} v_{1}(s)\right)-f\left(s, v_{2}(s),{ }^{c} D_{0^{+}}^{\alpha} v_{2}(s)\right)\right| d s\right. \\
& \left.+\left|\left(\mathcal{A} v_{1}\right)(u)-\left(\mathcal{A} v_{2}\right)(u)\right|\right) d u \\
& \left.\left.\leq \int_{0}^{t} \frac{(t-s)^{\beta-1}}{\Gamma(\beta)}\left[L_{1 f}\left|v_{1}(s)-v_{2}(s)\right|+L_{2 f} \mid{ }^{c} D_{0^{+}}^{\alpha} \nu_{1}(s)\right)-{ }^{c} D_{0^{+}}^{\alpha} v_{2}(s)\right) \mid\right] d s \\
& +L_{\mathcal{A}}\left|v_{1}(t)-v_{2}(t)\right|+\left|\frac{t \Gamma(\alpha+2)-\Gamma(\alpha+1) \eta_{1}}{\zeta_{1}^{\alpha+1}-\zeta_{1}^{\alpha} \eta_{1}}\right| \\
& \times \int_{0}^{\zeta_{1}} \frac{\left(\zeta_{1}-u\right)^{\alpha-1}}{\Gamma(\alpha)}\left(\int _ { 0 } ^ { u } \frac { ( u - s ) ^ { \beta - 1 } } { \Gamma ( \beta ) } \left[L_{1 f}\left|v_{1}(s)-v_{2}(s)\right|\right.\right. \\
& \left.\left.\left.\left.+L_{2 f} \mid{ }^{c} D_{0^{+}}^{\alpha} v_{1}(s)\right)-{ }^{c} D_{0^{+}}^{\alpha} \nu_{2}(s)\right) \mid\right] d s+L_{\mathcal{A}}\left|v_{1}(u)-v_{2}(u)\right|\right) d u \\
& +\left|\frac{\Gamma(\alpha+1) \zeta_{1}-t \Gamma(\alpha+2)}{\zeta_{1} \eta_{1}^{\alpha}-\eta_{1}^{\alpha+1}}\right| \int_{0}^{\eta_{1}} \frac{\left(\eta_{1}-u\right)^{\alpha-1}}{\Gamma(\alpha)}\left(\int_{0}^{u} \frac{(u-s)^{\beta-1}}{\Gamma(\beta)}\right. \\
& \left.\left.\times\left[L_{1 f}\left|v_{1}(s)-v_{2}(s)\right|+L_{2 f} \mid{ }^{c} D_{0^{+}}^{\alpha} v_{1}(s)\right)-{ }^{c} D_{0^{+}}^{\alpha} v_{2}(s)\right) \mid\right] d s \\
& \left.+L_{\mathcal{A}}\left|v_{1}(u)-v_{2}(u)\right|\right) d u \\
& \leq\left(\frac{\left(L_{1 f}+L_{2 f}\right) t^{\beta}}{\Gamma(\beta+1)}+L_{\mathcal{A}}\right)\left\|v_{1}-v_{2}\right\|_{X}+\left(\frac{\left(L_{1 f}+L_{2 f}\right) \zeta_{1}^{\alpha+\beta}}{\Gamma(\alpha+\beta+1)}\right. \\
& \left.+\frac{L_{\mathcal{A}} \zeta_{1}^{\alpha}}{\Gamma(\alpha+1)}\right) \frac{t_{1} \Gamma(\alpha+2)-\Gamma(\alpha+1) \eta_{1}}{\zeta_{1}^{\alpha+1}-\zeta_{1}^{\alpha} \eta_{1}}\left\|v_{1}-v_{2}\right\|_{X} \\
& +\left(\frac{\left(L_{1 f}+L_{2 f}\right) \eta_{1}^{\alpha+\beta}}{\Gamma(\alpha+\beta+1)}+\frac{L_{\mathcal{A}} \eta_{1}^{\alpha}}{\Gamma(\alpha+1)}\right) \frac{t_{1} \Gamma(\alpha+2)-\Gamma(\alpha+1) \zeta_{1}}{\eta_{1}^{\alpha} \zeta_{1}-\eta_{1}^{\alpha+1}}\left\|v_{1}-v_{2}\right\|_{X} \\
& \leq\left\{( L _ { 1 f } + L _ { 2 f } ) \left[\frac{t_{1}^{\beta}}{\Gamma(\beta+1)}+\frac{1}{\Gamma(\alpha+\beta+1)}\left(\frac{t_{1} \Gamma(\alpha+2)-\Gamma(\alpha+1) \eta_{1}}{\zeta_{1}^{\alpha+1}-\zeta_{1}^{\alpha} \eta_{1}} \zeta_{1}^{\alpha+\beta}\right.\right.\right. \\
& \left.\left.+\frac{t_{1} \Gamma(\alpha+2)-\Gamma(\alpha+1) \zeta_{1}}{\eta_{1}^{\alpha} \zeta_{1}-\eta_{1}^{\alpha+1}} \eta_{1}^{\alpha+\beta}\right)\right] \\
& +L_{\mathcal{A}}\left[1+\frac{1}{\Gamma(\alpha+1)}\left(\frac{t_{1} \Gamma(\alpha+2)-\Gamma(\alpha+1) \eta_{1}}{\zeta_{1}^{\alpha+1}-\zeta_{1}^{\alpha} \eta_{1}} \zeta_{1}^{\alpha}\right.\right. \\
& \left.\left.\left.+\frac{t_{1} \Gamma(\alpha+2)-\Gamma(\alpha+1) \zeta_{1}}{\eta_{1}^{\alpha} \zeta_{1}-\eta_{1}^{\alpha+1}} \eta_{1}^{\alpha}\right)\right]\right\}\left\|v_{1}-v_{2}\right\|_{X} \\
& =\mu_{0}\left\|v_{1}-v_{2}\right\|_{X}, \quad t \in\left[0, t_{1}\right] \\
& \left|Q\left(v_{1}\right)(t)-Q\left(v_{2}\right)(t)\right|=\left|g_{i}\left(t, v_{1}\left(t_{i}^{-}\right)\right)-g_{i}\left(t, v_{2}\left(t_{i}^{-}\right)\right)\right| \leq L_{g_{i}}\left|v_{1}\left(t_{i}^{-}\right)-v_{2}\left(t_{i}^{-}\right)\right| \\
& \leq L_{g_{i}}\left\|v_{1}-v_{2}\right\|_{X}, \quad t \in\left(t_{i}, s_{i}\right], i=1,2, \ldots, m, \\
& \left|Q\left(v_{1}\right)(t)-Q\left(v_{2}\right)(t)\right| \\
& \leq \int_{s_{i}}^{t} \frac{(t-u)^{\alpha-1}}{\Gamma(\alpha)}\left(\int_{s_{i}}^{u} \frac{(u-s)^{\beta-1}}{\Gamma(\beta)} \mid f\left(s, v_{1}(s),{ }^{c} D_{s_{i}^{+}}^{\alpha} v_{1}(s)\right)\right.
\end{aligned}
$$




$$
\begin{aligned}
& \left.-f\left(s, v_{2}(s),{ }^{c} D_{s_{i}^{+}}^{\alpha} v_{2}(s)\right)|d s+|\left(\mathcal{A} v_{1}\right)(u)-\left(\mathcal{A} v_{2}\right)(u) \mid\right) d u \\
& +\left|\mathcal{M}_{i}\right| t^{\alpha+1}\left[( \zeta _ { i + 1 } ^ { \alpha } - s _ { i } ^ { \alpha } ) \int _ { s _ { i } } ^ { \eta _ { i + 1 } } \frac { ( \eta _ { i + 1 } - u ) ^ { \alpha - 1 } } { \Gamma ( \alpha ) } \left(\int_{s_{i}}^{u} \frac{(u-s)^{\beta-1}}{\Gamma(\beta)}\right.\right. \\
& \times\left|f\left(s, v_{1}(s),{ }^{c} D_{s_{i}^{+}}^{\alpha} v_{1}(s)\right)-f\left(s, v_{2}(s),{ }^{c} D_{s_{i}^{+}}^{\alpha} v_{2}(s)\right)\right| d s \\
& \left.+\left|\left(\mathcal{A} v_{1}\right)(u)-\left(\mathcal{A} v_{2}\right)(u)\right|\right) d u+\left(\eta_{i+1}^{\alpha}-s_{i}^{\alpha}\right) \int_{s_{i}}^{\zeta_{i+1}} \frac{\left(\zeta_{i+1}-u\right)^{\alpha-1}}{\Gamma(\alpha)} \\
& \times\left(\int_{s_{i}}^{u} \frac{(u-s)^{\beta-1}}{\Gamma(\beta)}\left|f\left(s, v_{1}(s),{ }^{c} D_{s_{i}^{+}}^{\alpha} v_{1}(s)\right)-f\left(s, v_{2}(s),{ }^{c} D_{s_{i}^{+}}^{\alpha} v_{2}(s)\right)\right| d s\right. \\
& \left.\left.+\left|\left(\mathcal{A} v_{1}\right)(u)-\left(\mathcal{A} v_{2}\right)(u)\right|\right) d u+\left(\zeta_{i+1}^{\alpha}-\eta_{i+1}^{\alpha}\right)\left|g_{i}\left(s_{i}, v_{1}\left(t_{i}^{-}\right)\right)-g_{i}\left(s_{i}, v_{2}\left(t_{i}^{-}\right)\right)\right|\right] \\
& +\left|\mathcal{M}_{i}\right| t^{\alpha}\left[( \eta _ { i + 1 } ^ { \alpha + 1 } - s _ { i } ^ { \alpha + 1 } ) \int _ { s _ { i } } ^ { \zeta _ { i + 1 } } \frac { ( \zeta _ { i + 1 } - u ) ^ { \alpha - 1 } } { \Gamma ( \alpha ) } \left(\int_{s_{i}}^{u} \frac{(u-s)^{\beta-1}}{\Gamma(\beta)} \mid f\left(s, v_{1}(s),\right.\right.\right. \\
& \left.\left.{ }^{c} D_{s_{i}^{+}}^{\alpha} v_{1}(s)\right)-f\left(s, v_{2}(s),{ }^{c} D_{s_{i}^{+}}^{\alpha} v_{2}(s)\right)|d s+|\left(\mathcal{A} v_{1}\right)(u)-\left(\mathcal{A} v_{2}\right)(u) \mid\right) d u \\
& +\left(\zeta_{i+1}^{\alpha+1}-s_{i}^{\alpha+1}\right) \int_{s_{i}}^{\eta_{i+1}} \frac{\left(\eta_{i+1}-u\right)^{\alpha-1}}{\Gamma(\alpha)}\left(\int_{s_{i}}^{u} \frac{(u-s)^{\beta-1}}{\Gamma(\beta)} \mid f\left(s, v_{1}(s),{ }^{c} D_{s_{i}^{+}}^{\alpha} \nu_{1}(s)\right)\right. \\
& \left.-f\left(s, v_{2}(s),{ }^{c} D_{s_{i}^{+}}^{\alpha} v_{2}(s)\right)|d s+|\left(\mathcal{A} v_{1}\right)(u)-\left(\mathcal{A} v_{2}\right)(u) \mid\right) d u \\
& \left.+\left(\zeta_{i+1}^{\alpha+1}-\eta_{i+1}^{\alpha+1}\right)\left|g_{i}\left(s_{i}, v_{1}\left(t_{i}^{-}\right)\right)-g_{i}\left(s_{i}, v_{2}\left(t_{i}^{-}\right)\right)\right|\right] \\
& +\left|\mathcal{M}_{i}\right|\left[( \zeta _ { i + 1 } ^ { \alpha + 1 } s _ { i } ^ { \alpha } - s _ { i } ^ { \alpha + 1 } \zeta _ { i + 1 } ^ { \alpha } ) \int _ { s _ { i } } ^ { \eta _ { i + 1 } } \frac { ( \eta _ { i + 1 } - u ) ^ { \alpha - 1 } } { \Gamma ( \alpha ) } \left(\int_{s_{i}}^{u} \frac{(u-s)^{\beta-1}}{\Gamma(\beta)} \mid f\left(s, v_{1}(s),\right.\right.\right. \\
& \left.\left.{ }^{c} D_{s_{i}^{+}}^{\alpha} v_{1}(s)\right)-f\left(s, v_{2}(s),{ }^{c} D_{s_{i}^{+}}^{\alpha} v_{2}(s)\right)|d s+|\left(\mathcal{A} v_{1}\right)(u)-\left(\mathcal{A} v_{2}\right)(u) \mid\right) d u \\
& +\left(\eta_{i+1}^{\alpha+1} s_{i}^{\alpha}-s_{i}^{\alpha+1} \eta_{i+1}^{\alpha}\right) \int_{s_{i}}^{\zeta_{i+1}} \frac{\left(\zeta_{i+1}-u\right)^{\alpha-1}}{\Gamma(\alpha)}\left(\int_{s_{i}}^{u} \frac{(u-s)^{\beta-1}}{\Gamma(\beta)} \mid f\left(s, \nu_{1}(s),{ }^{c} D_{s_{i}^{+}}^{\alpha} v_{1}(s)\right)\right. \\
& \left.-f\left(s, v_{2}(s),{ }^{c} D_{s_{i}^{+}}^{\alpha} v_{2}(s)\right)|d s+|\left(\mathcal{A} v_{1}\right)(u)-\left(\mathcal{A} v_{2}\right)(u) \mid\right) d u \\
& \left.+\left(\zeta_{i+1}^{\alpha+1} \eta_{i+1}^{\alpha}-\eta_{i+1}^{\alpha+1} \zeta_{i+1}^{\alpha}\right)\left|g_{i}\left(s_{i}, v_{1}\left(t_{i}^{-}\right)\right)-g_{i}\left(s_{i}, v_{1}\left(t_{i}^{-}\right)\right)\right|\right] \\
& \leq \int_{0}^{t} \frac{(t-u)^{\alpha-1}}{\Gamma(\alpha)}\left(\int _ { 0 } ^ { u } \frac { ( u - s ) ^ { \beta - 1 } } { \Gamma ( \beta ) } \left[L_{1 f}\left|v_{1}(s)-v_{2}(s)\right|\right.\right. \\
& \left.+L_{2 f}\left|{ }^{c} D_{s_{i}^{+}}^{\alpha} v_{1}(s)-{ }^{c} D_{s_{i}^{+}}^{\alpha} v_{2}(s)\right| d s+L_{\mathcal{A}}\left|v_{1}(u)-v_{2}(u)\right|\right) d u \\
& +\left|\mathcal{M}_{i}\right| t^{\alpha+1}\left[( \zeta _ { i + 1 } ^ { \alpha } - s _ { i } ^ { \alpha } ) \int _ { 0 } ^ { \eta _ { i + 1 } } \frac { ( \eta _ { i + 1 } - u ) ^ { \alpha - 1 } } { \Gamma ( \alpha ) } \left(\int _ { 0 } ^ { u } \frac { ( u - s ) ^ { \beta - 1 } } { \Gamma ( \beta ) } \left[L_{1 f}\left|v_{1}(s)-v_{2}(s)\right|\right.\right.\right. \\
& \left.+L_{2 f}\left|{ }^{c} D_{s_{i}^{+}}^{\alpha} \nu_{1}(s)-{ }^{c} D_{s_{i}^{+}}^{\alpha} \nu_{2}(s)\right| d s+L_{\mathcal{A}}\left|v_{1}(u)-v_{2}(u)\right|\right) d u \\
& +\left(\eta_{i+1}^{\alpha}-s_{i}^{\alpha}\right) \int_{0}^{\zeta_{i+1}} \frac{\left(\zeta_{i+1}-u\right)^{\alpha-1}}{\Gamma(\alpha)}\left(\int _ { 0 } ^ { u } \frac { ( u - s ) ^ { \beta - 1 } } { \Gamma ( \beta ) } \left[L_{1 f}\left|v_{1}(s)-v_{2}(s)\right|\right.\right.
\end{aligned}
$$




$$
\begin{aligned}
& \left.+L_{2 f}\left|{ }^{c} D_{s_{i}^{+}}^{\alpha} v_{1}(s)-{ }^{c} D_{s_{i}^{+}}^{\alpha} v_{2}(s)\right| d s+L_{\mathcal{A}}\left|v_{1}(u)-v_{2}(u)\right|\right) d u \\
& \left.+\left(\zeta_{i+1}^{\alpha}-\eta_{i+1}^{\alpha}\right) L_{g_{i}}\left|v_{1}\left(t_{i}^{-}\right)-v_{2}\left(t_{i}^{-}\right)\right|\right] \\
& +\left|\mathcal{M}_{i}\right| t^{\alpha}\left[( \eta _ { i + 1 } ^ { \alpha + 1 } - s _ { i } ^ { \alpha + 1 } ) \int _ { 0 } ^ { \zeta _ { i + 1 } } \frac { ( \zeta _ { i + 1 } - u ) ^ { \alpha - 1 } } { \Gamma ( \alpha ) } \left(\int _ { 0 } ^ { u } \frac { ( u - s ) ^ { \beta - 1 } } { \Gamma ( \beta ) } \left[L_{1 f}\left|v_{1}(s)-v_{2}(s)\right|\right.\right.\right. \\
& \left.+L_{2 f}\left|{ }^{c} D_{s_{i}^{+}}^{\alpha} v_{1}(s)-{ }^{c} D_{s_{i}^{+}}^{\alpha} v_{2}(s)\right| d s+L_{\mathcal{A}}\left|v_{1}(u)-v_{2}(u)\right|\right) d u \\
& +\left(\zeta_{i+1}^{\alpha+1}-s_{i}^{\alpha+1}\right) \int_{0}^{\eta_{i+1}} \frac{\left(\eta_{i+1}-u\right)^{\alpha-1}}{\Gamma(\alpha)}\left(\int _ { 0 } ^ { u } \frac { ( u - s ) ^ { \beta - 1 } } { \Gamma ( \beta ) } \left[L_{1 f}\left|v_{1}(s)-v_{2}(s)\right|\right.\right. \\
& \left.+L_{2 f}\left|{ }^{c} D_{s_{i}^{+}}^{\alpha} v_{1}(s)-{ }^{c} D_{s_{i}^{+}}^{\alpha} v_{2}(s)\right| d s+L_{\mathcal{A}}\left|v_{1}(u)-v_{2}(u)\right|\right) d u \\
& \left.+\left(\zeta_{i+1}^{\alpha+1}-\eta_{i+1}^{\alpha+1}\right) L_{g_{i}}\left|v_{1}\left(t_{i}^{-}\right)-v_{2}\left(t_{i}^{-}\right)\right|\right] \\
& +\left|\mathcal{M}_{i}\right|\left[( \zeta _ { i + 1 } ^ { \alpha + 1 } s _ { i } ^ { \alpha } - s _ { i } ^ { \alpha + 1 } \zeta _ { i + 1 } ^ { \alpha } ) \int _ { 0 } ^ { \eta _ { i + 1 } } \frac { ( \eta _ { i + 1 } - u ) ^ { \alpha - 1 } } { \Gamma ( \alpha ) } \left(\int _ { 0 } ^ { u } \frac { ( u - s ) ^ { \beta - 1 } } { \Gamma ( \beta ) } \left[L_{1 f}\left|v_{1}(s)-v_{2}(s)\right|\right.\right.\right. \\
& \left.+L_{2 f}\left|{ }^{c} D_{s_{i}^{+}}^{\alpha} v_{1}(s)-{ }^{c} D_{s_{i}^{+}}^{\alpha} v_{2}(s)\right| d s+L_{\mathcal{A}}\left|v_{1}(u)-v_{2}(u)\right|\right) d u \\
& +\left(\eta_{i+1}^{\alpha+1} s_{i}^{\alpha}-s_{i}^{\alpha+1} \eta_{i+1}^{\alpha}\right) \int_{0}^{\zeta_{i+1}} \frac{\left(\zeta_{i+1}-u\right)^{\alpha-1}}{\Gamma(\alpha)}\left(\int _ { 0 } ^ { u } \frac { ( u - s ) ^ { \beta - 1 } } { \Gamma ( \beta ) } \left[L_{1 f}\left|v_{1}(s)-v_{2}(s)\right|\right.\right. \\
& \left.+L_{2 f}\left|{ }^{c} D_{s_{i}^{+}}^{\alpha} v_{1}(s)-{ }^{c} D_{s_{i}^{+}}^{\alpha} v_{2}(s)\right| d s+L_{\mathcal{A}}\left|v_{1}(u)-v_{2}(u)\right|\right) d u \\
& \left.+\left(\zeta_{i+1}^{\alpha+1} \eta_{i+1}^{\alpha}-\eta_{i+1}^{\alpha+1} \zeta_{i+1}^{\alpha}\right) L_{g_{i}}\left|v_{1}\left(t_{i}^{-}\right)-v_{1}\left(t_{i}^{-}\right)\right|\right] \\
& \leq\left\{\frac{L_{1 f}+L_{2 f}}{\Gamma(\alpha+\beta+1)} t^{\alpha+\beta}+\frac{L_{\mathcal{A}} t^{\alpha}}{\Gamma(\alpha+1)}+\left|\mathcal{M}_{i}\right| t^{\alpha+1}\left[( \zeta _ { i + 1 } ^ { \alpha } - s _ { i } ^ { \alpha } ) \left(\frac{L_{1 f}+L_{2 f}}{\Gamma(\alpha+\beta+1)} \eta_{i+1}^{\alpha+\beta}\right.\right.\right. \\
& \left.\left.+\frac{L_{\mathcal{A}} \eta_{i+1}^{\alpha}}{\Gamma(\alpha+1)}\right)+\left(\eta_{i+1}^{\alpha}-s_{i}^{\alpha}\right)\left(\frac{L_{1 f}+L_{2 f}}{\Gamma(\alpha+\beta+1)} \zeta_{i+1}^{\alpha+\beta}+\frac{L_{\mathcal{A}} \zeta_{i+1}^{\alpha}}{\Gamma(\alpha+1)}\right)+\left(\zeta_{i+1}^{\alpha}-\eta_{i+1}^{\alpha}\right) L_{g_{i}}\right] \\
& +\left|\mathcal{M}_{i}\right| t^{\alpha}\left[\left(\eta_{i+1}^{\alpha+1}-s_{i}^{\alpha+1}\right)\left(\frac{L_{1 f}+L_{2 f}}{\Gamma(\alpha+\beta+1)} \zeta_{i+1}^{\alpha+\beta}+\frac{L_{\mathcal{A}} \zeta_{i+1}^{\alpha}}{\Gamma(\alpha+1)}\right)\right. \\
& \left.+\left(\zeta_{i+1}^{\alpha+1}-s_{i}^{\alpha+1}\right)\left(\frac{L_{1 f}+L_{2 f}}{\Gamma(\alpha+\beta+1)} \eta_{i+1}^{\alpha+\beta}+\frac{L_{\mathcal{A}} \eta_{i+1}^{\alpha}}{\Gamma(\alpha+1)}\right)+\left(\zeta_{i+1}^{\alpha+1}-\eta_{i+1}^{\alpha+1}\right) L_{g_{i}}\right] \\
& +\left|\mathcal{M}_{i}\right|\left[\left(\zeta_{i+1}^{\alpha+1} s_{i}^{\alpha}-s_{i}^{\alpha+1} \zeta_{i+1}^{\alpha}\right)\left(\frac{L_{1 f}+L_{2 f}}{\Gamma(\alpha+\beta+1)} \eta_{i+1}^{\alpha+\beta}+\frac{L_{\mathcal{A}} \eta_{i+1}^{\alpha}}{\Gamma(\alpha+1)}\right)\right. \\
& +\left(\eta_{i+1}^{\alpha+1} s_{i}^{\alpha}-s_{i}^{\alpha+1} \eta_{i+1}^{\alpha}\right)\left(\frac{L_{1 f}+L_{2 f}}{\Gamma(\alpha+\beta+1)} \zeta_{i+1}^{\alpha+\beta}+\frac{L_{\mathcal{A}} \zeta_{i+1}^{\alpha}}{\Gamma(\alpha+1)}\right) \\
& \left.\left.+\left(\zeta_{i+1}^{\alpha+1} \eta_{i+1}^{\alpha}-\eta_{i+1}^{\alpha+1} \zeta_{i+1}^{\alpha}\right) L_{g_{i}}\right]\right\}\left\|v_{1}-v_{2}\right\|_{X} \\
& \leq\left\{\frac { L _ { 1 f } + L _ { 2 f } } { \Gamma ( \alpha + \beta + 1 ) } \left[t_{i+1}^{\alpha+\beta}+\left|\mathcal{M}_{i}\right| t_{i+1}^{\alpha+1}\left[\left(\zeta_{i+1}^{\alpha}-s_{i}^{\alpha}\right) \eta_{i+1}^{\alpha+\beta}+\left(\eta_{i+1}^{\alpha}-s_{i}^{\alpha}\right) \zeta_{i+1}^{\alpha+\beta}\right]\right.\right. \\
& +\left|\mathcal{M}_{i}\right| t_{i+1}^{\alpha}\left[\left(\eta_{i+1}^{\alpha+1}-s_{i}^{\alpha+1}\right) \zeta_{i+1}^{\alpha+\beta}+\left(\zeta_{i+1}^{\alpha+1}-s_{i}^{\alpha+1}\right) \eta_{i+1}^{\alpha+\beta}\right]+\left|\mathcal{M}_{i}\right|\left[\left(\zeta_{i+1}^{\alpha+1} s_{i}^{\alpha}\right.\right. \\
& \left.\left.\left.-s_{i}^{\alpha+1} \zeta_{i+1}^{\alpha}\right) \eta_{i+1}^{\alpha+\beta}+\left(\eta_{i+1}^{\alpha+1} s_{i}^{\alpha}-s_{i}^{\alpha+1} \eta_{i+1}^{\alpha}\right) \zeta_{i+1}^{\alpha+\beta}\right]\right]+\frac{L_{\mathcal{A}}}{\Gamma(\alpha+1)}\left[t_{i+1}^{\alpha}\right. \\
& +\left|\mathcal{M}_{i}\right| t_{i+1}^{\alpha+1}\left[\left(\zeta_{i+1}^{\alpha}-s_{i}^{\alpha}\right) \eta_{i+1}^{\alpha}+\left(\eta_{i+1}^{\alpha}-s_{i}^{\alpha}\right) \zeta_{i+1}^{\alpha}\right]+\left|\mathcal{M}_{i}\right| t_{i+1}^{\alpha}\left[\left(\eta_{i+1}^{\alpha}-s_{i}^{\alpha}\right) \zeta_{i+1}^{\alpha}\right.
\end{aligned}
$$




$$
\begin{aligned}
& \left.+\left(\zeta_{i+1}^{\alpha+1}-s_{i}^{\alpha+1}\right) \eta_{i+1}^{\alpha}\right]+\left|\mathcal{M}_{i}\right|\left[\left(\zeta_{i+1}^{\alpha+1} s_{i}^{\alpha}-s_{i}^{\alpha+1} \zeta_{i+1}^{\alpha}\right) \eta_{i+1}^{\alpha}+\left(\eta_{i+1}^{\alpha+1} s_{i}^{\alpha}\right.\right. \\
& \left.\left.\left.-s_{i}^{\alpha+1} \eta_{i+1}^{\alpha}\right) \zeta_{i+1}^{\alpha}\right]\right]+L_{g_{i}} \mathcal{M}_{i}\left[t_{i+1}^{\alpha+1}\left(\zeta_{i+1}^{\alpha}-\eta_{i+1}^{\alpha}\right)+t_{i+1}^{\alpha}\left(\zeta_{i+1}^{\alpha+1}-\eta_{i+1}^{\alpha+1}\right)\right. \\
& \left.\left.+\left(\zeta_{i+1}^{\alpha+1} \eta_{i+1}^{\alpha}-\eta_{i+1}^{\alpha+1} \zeta_{i+1}^{\alpha}\right)\right]\right\}\left\|v_{1}-v_{2}\right\|_{X} \\
& =\lambda_{i}\left\|v_{1}-v_{2}\right\|_{X}, \quad t \in\left(s_{i}, t_{i+1}\right], i=1,2, \ldots, m, \\
& \left|{ }^{c} D_{s_{i}^{+}}^{\alpha}\left(Q v_{1}\right)(t)-{ }^{c} D_{s_{i}^{+}}^{\alpha}\left(Q v_{2}\right)(t)\right| \\
& \leq \int_{s_{i}}^{t} \frac{(t-s)^{\beta-1}}{\Gamma(\beta)}\left|f\left(s, v_{1}(s),{ }^{c} D_{s_{i}^{+}}^{\alpha} v_{1}(s)\right)-f\left(s, v_{2}(s),{ }^{c} D_{s_{i}^{+}}^{\alpha} v_{2}(s)\right)\right| d s \\
& +\left|\left(\mathcal{A} v_{1}\right)(t)-\left(\mathcal{A} v_{2}\right)(t)\right|+\frac{\left|\mathcal{M}_{i}\right|}{\Gamma(1-\alpha)} \int_{s_{i}}^{t}(t-s)^{-\alpha}\left(s^{\alpha+1}\right)^{\prime} d s \\
& \times\left[( \zeta _ { i + 1 } ^ { \alpha } - s _ { i } ^ { \alpha } ) \int _ { s _ { i } } ^ { \eta _ { i + 1 } } \frac { ( \eta _ { i + 1 } - u ) ^ { \alpha - 1 } } { \Gamma ( \alpha ) } \left(\int_{s_{i}}^{u} \frac{(u-s)^{\beta-1}}{\Gamma(\beta)} \mid f\left(s, v_{1}(s),{ }^{c} D_{s_{i}^{+}}^{\alpha} v_{1}(s)\right)\right.\right. \\
& \left.-f\left(s, v_{2}(s),{ }^{c} D_{s_{i}^{+}}^{\alpha} v_{2}(s)\right)|d s+|\left(\mathcal{A} v_{1}\right)(u)-\left(\mathcal{A} v_{2}\right)(u) \mid\right) d u \\
& +\left(\eta_{i+1}^{\alpha}-s_{i}^{\alpha}\right) \int_{s_{i}}^{\zeta_{i+1}} \frac{\left(\zeta_{i+1}-u\right)^{\alpha-1}}{\Gamma(\alpha)}\left(\int_{s_{i}}^{u} \frac{(u-s)^{\beta-1}}{\Gamma(\beta)} \mid f\left(s, v_{1}(s),{ }^{c} D_{s_{i}^{+}}^{\alpha} \nu_{1}(s)\right)\right. \\
& \left.-f\left(s, v_{2}(s),{ }^{c} D_{s_{i}^{+}}^{\alpha} v_{2}(s)\right)|d s+|\left(\mathcal{A} v_{1}\right)(u)-\left(\mathcal{A} v_{2}\right)(u) \mid\right) d u \\
& \left.+\left(\zeta_{i+1}^{\alpha}-\eta_{i+1}^{\alpha}\right)\left|g_{i}\left(s_{i}, v_{1}\left(t_{i}^{-}\right)\right)-g_{i}\left(s_{i}, v_{2}\left(t_{i}^{-}\right)\right)\right|\right]+\frac{\left|\mathcal{M}_{i}\right|}{\Gamma(1-\alpha)} \int_{s_{i}}^{t}(t-s)^{-\alpha}\left(s^{\alpha}\right)^{\prime} d s \\
& \times\left[( \eta _ { i + 1 } ^ { \alpha + 1 } - s _ { i } ^ { \alpha + 1 } ) \int _ { s _ { i } } ^ { \zeta _ { i + 1 } } \frac { ( \zeta _ { i + 1 } - u ) ^ { \alpha - 1 } } { \Gamma ( \alpha ) } \left(\int_{s_{i}}^{u} \frac{(u-s)^{\beta-1}}{\Gamma(\beta)} \mid f\left(s, v_{1}(s),{ }^{c} D_{s_{i}^{+}}^{\alpha} \nu_{1}(s)\right)\right.\right. \\
& \left.-f\left(s, v_{2}(s),{ }^{c} D_{s_{i}^{+}}^{\alpha} v_{2}(s)\right)|d s+|\left(\mathcal{A} v_{1}\right)(u)-\left(\mathcal{A} v_{2}\right)(u) \mid\right) d u \\
& +\left(\zeta_{i+1}^{\alpha+1}-s_{i}^{\alpha+1}\right) \int_{s_{i}}^{\eta_{i+1}} \frac{\left(\eta_{i+1}-u\right)^{\alpha-1}}{\Gamma(\alpha)}\left(\int_{s_{i}}^{u} \frac{(u-s)^{\beta-1}}{\Gamma(\beta)} \mid f\left(s, v_{1}(s),{ }^{c} D_{s_{i}^{+}}^{\alpha} v_{1}(s)\right)\right. \\
& \left.-f\left(s, v_{2}(s),{ }^{c} D_{s_{i}^{+}}^{\gamma} v_{2}(s)\right)|d s+|\left(\mathcal{A} v_{1}\right)(u)-\left(\mathcal{A} v_{2}\right)(u) \mid\right) d u \\
& \left.+\left(\zeta_{i+1}^{\alpha+1}-\eta_{i+1}^{\alpha+1}\right)\left|g_{i}\left(s_{i}, v_{1}\left(t_{i}^{-}\right)\right)-g_{i}\left(s_{i}, v_{2}\left(t_{i}^{-}\right)\right)\right|\right] \\
& \left.\left.\leq \int_{0}^{t} \frac{(t-s)^{\beta-1}}{\Gamma(\beta)}\left[L_{1 f}\left|v_{1}(s)-v_{2}(s)\right|+L_{2 f} \mid{ }^{c} D_{0^{+}}^{\alpha} v_{1}(s)\right)-{ }^{c} D_{0^{+}}^{\alpha} v_{2}(s)\right) \mid\right] d s \\
& +L_{\mathcal{A}}\left|v_{1}(t)-v_{2}(t)\right|+\left|\mathcal{M}_{i}\right| t \Gamma(\alpha+2)\left[\left(\zeta_{i+1}^{\alpha}-s_{i}^{\alpha}\right) \int_{0}^{\eta_{i+1}} \frac{\left(\eta_{i+1}-u\right)^{\alpha-1}}{\Gamma(\alpha)}\right. \\
& \left.\times\left(\int_{0}^{u} \frac{(u-s)^{\beta-1}}{\Gamma(\beta)}\left[L_{1 f}\left|v_{1}(s)-v_{2}(s)\right|+L_{2 f} \mid{ }^{c} D_{0^{+}}^{\alpha} v_{1}(s)\right)-{ }^{c} D_{0^{+}}^{\alpha} v_{2}(s)\right) \mid\right] d s \\
& \left.+L_{\mathcal{A}}\left|v_{1}(u)-v_{2}(u)\right|\right) d u+\left(\eta_{i+1}^{\alpha}-s_{i}^{\alpha}\right) \int_{0}^{\zeta_{i+1}} \frac{\left(\zeta_{i+1}-u\right)^{\alpha-1}}{\Gamma(\alpha)}\left(\int_{0}^{u} \frac{(u-s)^{\beta-1}}{\Gamma(\beta)}\right. \\
& \left.\left.\left.\times\left[L_{1 f}\left|v_{1}(s)-v_{2}(s)\right|+L_{2 f} \mid{ }^{c} D_{0^{+}}^{\alpha} v_{1}(s)\right)-{ }^{c} D_{0^{+}}^{\alpha} v_{2}(s)\right) \mid\right] d s+L_{\mathcal{A}}\left|v_{1}(u)-v_{2}(u)\right|\right) d u
\end{aligned}
$$




$$
\begin{aligned}
& \left.+\left(\zeta_{i+1}^{\alpha}-\eta_{i+1}^{\alpha}\right) L_{g_{i}}\left|v_{1}\left(t_{i}^{-}\right)-v_{2}\left(t_{i}^{-}\right)\right|\right]+\left|\mathcal{M}_{i}\right| \Gamma(\alpha+1)\left[\left(\eta_{i+1}^{\alpha+1}-s_{i}^{\alpha+1}\right)\right. \\
& \times \int_{0}^{\zeta_{i+1}} \frac{\left(\zeta_{i+1}-u\right)^{\alpha-1}}{\Gamma(\alpha)}\left(\int _ { 0 } ^ { u } \frac { ( u - s ) ^ { \beta - 1 } } { \Gamma ( \beta ) } \left[L_{1 f}\left|v_{1}(s)-v_{2}(s)\right|\right.\right. \\
& \left.\left.\left.\left.+L_{2 f} \mid{ }^{c} D_{0^{+}}^{\alpha} v_{1}(s)\right)-{ }^{c} D_{0^{+}}^{\alpha} \nu_{2}(s)\right) \mid\right] d s+L_{\mathcal{A}}\left|v_{1}(u)-v_{2}(u)\right|\right) d u \\
& +\left(\zeta_{i+1}^{\alpha+1}-s_{i}^{\alpha+1}\right) \int_{0}^{\eta_{i+1}} \frac{\left(\eta_{i+1}-u\right)^{\alpha-1}}{\Gamma(\alpha)}\left(\int _ { 0 } ^ { u } \frac { ( u - s ) ^ { \beta - 1 } } { \Gamma ( \beta ) } \left[L_{1 f}\left|v_{1}(s)-v_{2}(s)\right|\right.\right. \\
& \left.\left.\left.\left.+L_{2 f} \mid{ }^{c} D_{0^{+}}^{\alpha} v_{1}(s)\right)-{ }^{c} D_{0^{+}}^{\alpha} \nu_{2}(s)\right) \mid\right] d s+L_{\mathcal{A}}\left|v_{1}(u)-v_{2}(u)\right|\right) d u \\
& \left.+\left(\zeta_{i+1}^{\alpha+1}-\eta_{i+1}^{\alpha+1}\right) L_{g_{i}}\left|v_{1}\left(t_{i}^{-}\right)-v_{2}\left(t_{i}^{-}\right)\right|\right] \\
& \leq\left\{\frac{\left(L_{1 f}+L_{2 f}\right) t^{\beta}}{\Gamma(\beta+1)}+L_{\mathcal{A}}+\left|\mathcal{M}_{i}\right| t_{i+1} \Gamma(\alpha+2)\left[\frac{\left(\zeta_{i+1}^{\alpha}-s_{i}^{\alpha}\right)\left(L_{1 f}+L_{2 f}\right) \eta_{i+1}^{\alpha+\beta}}{\Gamma(\alpha+\beta+1)}\right.\right. \\
& +\frac{\left(\zeta_{i+1}^{\alpha}-s_{i}^{\alpha}\right) L_{\mathcal{A}} \eta_{i+1}^{\alpha}}{\Gamma(\alpha+1)}+\frac{\left(\eta_{i+1}^{\alpha}-s_{i}^{\alpha}\right)\left(L_{1 f}+L_{2 f}\right) \zeta_{i+1}^{\alpha+\beta}}{\Gamma(\alpha+\beta+1)}+\frac{\left(\eta_{i+1}^{\alpha}-s_{i}^{\alpha}\right) L_{\mathcal{A}} \zeta_{i+1}^{\alpha}}{\Gamma(\alpha+1)} \\
& \left.+\left(\zeta_{i+1}^{\alpha}-\eta_{i+1}^{\alpha}\right) L_{g_{i}}\right]+\left|\mathcal{M}_{i}\right| \Gamma(\alpha+1)\left[\frac{\left(\eta_{i+1}^{\alpha+1}-s_{i}^{\alpha+1}\right)\left(L_{1 f}+L_{2 f}\right) \zeta_{i+1}^{\alpha+\beta}}{\Gamma(\alpha+\beta+1)}\right. \\
& +\frac{\left(\eta_{i+1}^{\alpha+1}-s_{i}^{\alpha+1}\right) L_{\mathcal{A}} \zeta_{i+1}^{\alpha}}{\Gamma(\alpha+1)}+\frac{\left(\zeta_{i+1}^{\alpha+1}-s_{i}^{\alpha+1}\right)\left(L_{1 f}+L_{2 f}\right) \eta_{i+1}^{\alpha+\beta}}{\Gamma(\alpha+\beta+1)} \\
& \left.\left.+\frac{\left(\zeta_{i+1}^{\alpha+1}-s_{i}^{\alpha+1}\right) L_{\mathcal{A}} \eta_{i+1}^{\alpha}}{\Gamma(\alpha+1)}+\left(\zeta_{i+1}^{\alpha+1}-\eta_{i+1}^{\alpha+1}\right) L_{g_{i}}\right]\right\}\left\|v_{1}-v_{2}\right\|_{X} \\
& \leq\left\{( L _ { 1 f } + L _ { 2 f } ) \left(\frac{t_{i+1}^{\beta}}{\Gamma(\beta+1)}+\frac{\left|\mathcal{M}_{i}\right| t_{i+1} \Gamma(\alpha+2)}{\Gamma(\alpha+\beta+1)}\left[\left(\zeta_{i+1}^{\alpha}-s_{i}^{\alpha}\right) \eta_{i+1}^{\alpha+\beta}+\left(\eta_{i+1}^{\alpha}-s_{i}^{\alpha}\right) \zeta_{i+1}^{\alpha+\beta}\right]\right.\right. \\
& \left.+\frac{\left|\mathcal{M}_{i}\right|}{\Gamma(\alpha+\beta+1)}\left[\left(\zeta_{i+1}^{\alpha+1}-s_{i}^{\alpha+1}\right) \eta_{i+1}^{\alpha+\beta}+\left(\eta_{i+1}^{\alpha+1}-s_{i}^{\alpha+1}\right) \zeta_{i+1}^{\alpha+\beta}\right]\right) \\
& +L_{\mathcal{A}}\left(1+\left|\mathcal{M}_{i}\right| t_{i+1}(\alpha+1)\left[\left(\zeta_{i+1}^{\alpha}-s_{i}^{\alpha}\right) \eta_{i+1}^{\alpha}+\left(\eta_{i+1}^{\alpha}-s_{i}^{\alpha}\right) \zeta_{i+1}^{\alpha}\right]\right. \\
& \left.+\left|\mathcal{M}_{i}\right|\left[\left(\zeta_{i+1}^{\alpha+1}-s_{i}^{\alpha+1}\right) \eta_{i+1}^{\alpha}+\left(\eta_{i+1}^{\alpha+1}-s_{i}^{\alpha+1}\right) \zeta_{i+1}^{\alpha}\right]\right) \\
& \left.+L_{g_{i}}\left|\mathcal{M}_{i}\right|\left[t_{i+1} \Gamma(\alpha+2)\left(\zeta_{i+1}^{\alpha}-\eta_{i+1}^{\alpha}\right)+\Gamma(\alpha+1)\left(\zeta_{i+1}^{\alpha+1}-\eta_{i+1}^{\alpha+1}\right)\right]\right\}\left\|v_{1}-v_{2}\right\|_{X} \\
& =\mu_{i}\left\|v_{1}-v_{2}\right\|_{X}, \quad t \in\left(s_{i}, t_{i+1}\right], i=1,2, \ldots, m .
\end{aligned}
$$

It follows from (3.3)-(3.7) that

$$
\left\|\left(Q v_{1}\right)(t)-\left(Q v_{2}\right)(t)\right\|_{X} \leq \lambda\left\|v_{1}-v_{2}\right\|_{X}
$$

According to $\left(H_{4}\right)$ and (3.8), we know that $Q$ is strictly contractive on $X$. In the light of the Banach contraction principle, we conclude that the problem (1.1) has a unique solution $y_{0} \in X$ and satisfies the integral equation (2.1).

Next we show that (2) of Theorem 3.1 also holds. Indeed, let $y \in X$ be a solution of the inequality (2.10) and $y_{0} \in X$ be a unique solution of (1.1). According to Lemma 2.3 and 
(3.2), we have

$$
\left\{\begin{array}{l}
\left|y(t)-y_{0}(t)\right| \leq \kappa_{0} \varepsilon, \quad t \in\left[0, t_{1}\right], \\
\left|y(t)-y_{0}(t)\right| \leq \varepsilon, \quad t \in\left(t_{i}, s_{i}\right], i=1,2, \ldots, m, \\
\left|y(t)-y_{0}(t)\right| \leq \kappa_{i} \varepsilon, \quad t \in\left(s_{i}, t_{i+1}\right], i=1,2, \ldots, m .
\end{array}\right.
$$

Then (3.9) gives that $\left|y(t)-y_{0}(t)\right| \leq \kappa \varepsilon, t \in J$, namely, the problem (1.1) is Ulam-Hyers stable. The proof of Theorem 3.1 is completed.

\section{Illustrative example}

Consider the fractional-order six-point boundary value problem involving noninstantaneous impulse and bounded operator as follows:

$$
\left\{\begin{array}{l}
{ }^{c} D_{s_{i}^{+}}^{\frac{3}{2}}\left({ }^{c} D_{s_{i}^{+}}^{\frac{1}{2}}+\mathcal{A}\right) x(t)=\frac{x(t)+{ }^{c} D_{s_{i}^{+}}^{\frac{1}{2}} x(t)}{(t+20)^{2}\left(1+(x(t))^{2}+\left({ }^{c} D_{s_{i}^{+}}^{\frac{1}{2}} x(t)\right)^{2}\right)}, \quad t \in\left[0, \frac{1}{3}\right] \cup\left(\frac{2}{3}, 1\right], \\
x(t)=\frac{x\left(t_{1}^{-}\right)}{80(1+t)^{2}}, \quad t \in\left(\frac{1}{3}, \frac{2}{3}\right], \\
x(0)=x\left(\frac{1}{5}\right)=x\left(\frac{1}{4}\right)=x\left(\frac{3}{4}\right)=x\left(\frac{11}{12}\right)=x(1)=0 .
\end{array}\right.
$$

Obviously, $\alpha=\frac{1}{2}, \beta=\frac{3}{2}, 0=s_{0}<\eta_{1}=\frac{1}{5}<\zeta_{1}=\frac{1}{4}<t_{1}=\frac{1}{3}<s_{1}=\frac{2}{3}<\eta_{2}=\frac{3}{4}<\zeta_{2}=\frac{11}{12}<t_{2}=$ $1, f\left(t, x(t), D_{s_{i}^{+}}^{\alpha} x(t)\right)=\frac{x(t)+{ }^{c} D_{s_{i}^{+}}^{\alpha} x(t)}{(t+20)^{2}\left(1+(x(t))^{2}+\left({ }^{c} D_{s_{i}^{+}}^{\alpha} x(t)\right)^{2}\right)}$ with $L_{1 f}=L_{1 f}=\frac{1}{400}, g_{1}(t, x(t))=\frac{x\left(t_{1}^{-}\right)}{80(1+t)^{2}}$ with $L_{g_{1}}=\frac{1}{320}<1$. Let $(\mathcal{A} x)(t)=\frac{x(t)}{320}$, then $L_{\mathcal{A}}=\frac{1}{320}$. So the conditions $\left(H_{1}\right)-\left(H_{3}\right)$ hold. Next we verify that $\left(H_{4}\right)$ holds. Indeed,

$$
\begin{aligned}
& \mathcal{M}_{1}=\frac{1}{\left(\eta_{2}^{\alpha+1}-s_{1}^{\alpha+1}\right)\left(\zeta_{2}^{\alpha}-s_{1}^{\alpha}\right)-\left(\zeta_{2}^{\alpha}-s_{1}^{\alpha}\right)\left(\eta_{2}^{\alpha}-s_{1}^{\alpha}\right)} \approx 127.4847, \\
& \lambda_{0}=\frac{L_{1 f}+L_{2 f}}{\Gamma(\alpha+\beta+1)}\left(t_{1}^{\alpha+\beta}+\frac{t_{1}^{\alpha+1}-t_{1}^{\alpha} \eta_{1}}{\zeta_{1}^{\alpha+1}-\zeta_{1}^{\alpha} \eta_{1}} \zeta_{1}^{\alpha+\beta}+\frac{t_{1}^{\alpha+1}-t_{1}^{\alpha} \zeta_{1}}{\eta_{1}^{\alpha} \zeta_{1}-\eta_{1}^{\alpha+1}} \eta_{1}^{\alpha+\beta}\right) \\
& +\frac{L_{\mathcal{A}}}{\Gamma(\alpha+1)}\left(t_{1}^{\alpha}+\frac{t_{1}^{\alpha+1}-t_{1}^{\alpha} \eta_{1}}{\zeta_{1}^{\alpha+1}-\zeta_{1}^{\alpha} \eta_{1}} \zeta_{1}^{\alpha}+\frac{t_{1}^{\alpha+1}-t_{1}^{\alpha} \zeta_{1}}{\eta_{1}^{\alpha} \zeta_{1}-\eta_{1}^{\alpha+1}} \eta_{1}^{\alpha}\right) \approx 0.0119<1, \\
& \mu_{0}=\left(L_{1 f}+L_{2 f}\right)\left[\frac{t_{1}^{\beta}}{\Gamma(\beta+1)}+\frac{1}{\Gamma(\alpha+\beta+1)}\left(\frac{t_{1} \Gamma(\alpha+2)-\Gamma(\alpha+1) \eta_{1}}{\zeta_{1}^{\alpha+1}-\zeta_{1}^{\alpha} \eta_{1}} \zeta_{1}^{\alpha+\beta}\right.\right. \\
& \left.\left.+\frac{t_{1} \Gamma(\alpha+2)-\Gamma(\alpha+1) \zeta_{1}}{\eta_{1}^{\alpha} \zeta_{1}-\eta_{1}^{\alpha+1}} \eta_{1}^{\alpha+\beta}\right)\right]+L_{\mathcal{A}}\left[1+\frac{1}{\Gamma(\alpha+1)}\right. \\
& \left.\times\left(\frac{t_{1} \Gamma(\alpha+2)-\Gamma(\alpha+1) \eta_{1}}{\zeta_{1}^{\alpha+1}-\zeta_{1}^{\alpha} \eta_{1}} \zeta_{1}^{\alpha}+\frac{t_{1} \Gamma(\alpha+2)-\Gamma(\alpha+1) \zeta_{1}}{\eta_{1}^{\alpha} \zeta_{1}-\eta_{1}^{\alpha+1}} \eta_{1}^{\alpha}\right)\right] \approx 0.0409<1 \text {, } \\
& \lambda_{1}=\frac{L_{1 f}+L_{2 f}}{\Gamma(\alpha+\beta+1)}\left[t_{2}^{\alpha+\beta}+\left|\mathcal{M}_{1}\right| t_{2}^{\alpha+1}\left[\left(\zeta_{2}^{\alpha}-s_{1}^{\alpha}\right) \eta_{2}^{\alpha+\beta}+\left(\eta_{2}^{\alpha}-s_{1}^{\alpha}\right) \zeta_{2}^{\alpha+\beta}\right]\right. \\
& +\left|\mathcal{M}_{1}\right| t_{2}^{\alpha}\left[\left(\eta_{2}^{\alpha+1}-s_{1}^{\alpha+1}\right) \zeta_{2}^{\alpha+\beta}+\left(\zeta_{2}^{\alpha+1}-s_{1}^{\alpha+1}\right) \eta_{2}^{\alpha+\beta}\right]+\left|\mathcal{M}_{1}\right|\left[\left(\zeta_{2}^{\alpha+1} s_{1}^{\alpha}\right.\right. \\
& \left.\left.\left.-s_{1}^{\alpha+1} \zeta_{2}^{\alpha}\right) \eta_{2}^{\alpha+\beta}+\left(\eta_{2}^{\alpha+1} s_{1}^{\alpha}-s_{1}^{\alpha+1} \eta_{2}^{\alpha}\right) \zeta_{2}^{\alpha+\beta}\right]\right]+\frac{L_{\mathcal{A}}}{\Gamma(\alpha+1)}\left[t_{2}^{\alpha}\right. \\
& +\left|\mathcal{M}_{1}\right| t_{2}^{\alpha+1}\left[\left(\zeta_{2}^{\alpha}-s_{1}^{\alpha}\right) \eta_{2}^{\alpha}+\left(\eta_{2}^{\alpha}-s_{1}^{\alpha}\right) \zeta_{2}^{\alpha}\right]+\left|\mathcal{M}_{1}\right| t_{2}^{\alpha}\left[\left(\eta_{2}^{\alpha}-s_{1}^{\alpha}\right) \zeta_{2}^{\alpha}\right. \\
& \left.+\left(\zeta_{2}^{\alpha+1}-s_{1}^{\alpha+1}\right) \eta_{2}^{\alpha}\right]+\left|\mathcal{M}_{1}\right|\left[\left(\zeta_{2}^{\alpha+1} s_{1}^{\alpha}-s_{1}^{\alpha+1} \zeta_{2}^{\alpha}\right) \eta_{2}^{\alpha}+\left(\eta_{2}^{\alpha+1} s_{1}^{\alpha}\right.\right.
\end{aligned}
$$




$$
\begin{aligned}
& \left.\left.\left.-s_{1}^{\alpha+1} \eta_{2}^{\alpha}\right) \zeta_{2}^{\alpha}\right]\right]+L_{g_{1}} \mathcal{M}_{1}\left[t_{2}^{\alpha+1}\left(\zeta_{2}^{\alpha}-\eta_{2}^{\alpha}\right)+t_{2}^{\alpha}\left(\zeta_{2}^{\alpha+1}-\eta_{2}^{\alpha+1}\right)\right. \\
& \left.+\left(\zeta_{2}^{\alpha+1} \eta_{2}^{\alpha}-\eta_{2}^{\alpha+1} \zeta_{2}^{\alpha}\right)\right] \approx 0.7570<1, \\
\mu_{1}= & \left(L_{1 f}+L_{2 f}\right)\left(\frac{t_{2}^{\beta}}{\Gamma(\beta+1)}+\frac{\left|\mathcal{M}_{1}\right| t_{2} \Gamma(\alpha+2)}{\Gamma(\alpha+\beta+1)}\left[\left(\zeta_{2}^{\alpha}-s_{1}^{\alpha}\right) \eta_{2}^{\alpha+\beta}+\left(\eta_{2}^{\alpha}-s_{1}^{\alpha}\right) \zeta_{2}^{\alpha+\beta}\right]\right. \\
& \left.+\frac{\left|\mathcal{M}_{1}\right|}{\Gamma(\alpha+\beta+1)}\left[\left(\zeta_{2}^{\alpha+1}-s_{1}^{\alpha+1}\right) \eta_{2}^{\alpha+\beta}+\left(\eta_{2}^{\alpha+1}-s_{1}^{\alpha+1}\right) \zeta_{2}^{\alpha+\beta}\right]\right) \\
& +L_{\mathcal{A}}\left(1+\left|\mathcal{M}_{1}\right| t_{2}(\alpha+1)\left[\left(\zeta_{2}^{\alpha}-s_{1}^{\alpha}\right) \eta_{2}^{\alpha}+\left(\eta_{2}^{\alpha}-s_{1}^{\alpha}\right) \zeta_{2}^{\alpha}\right]\right. \\
& \left.+\left|\mathcal{M}_{1}\right|\left[\left(\zeta_{2}^{\alpha+1}-s_{1}^{\alpha+1}\right) \eta_{2}^{\alpha}+\left(\eta_{2}^{\alpha+1}-s_{1}^{\alpha+1}\right) \zeta_{2}^{\alpha}\right]\right) \\
& +L_{g_{1}}\left|\mathcal{M}_{1}\right|\left[t_{2} \Gamma(\alpha+2)\left(\zeta_{2}^{\alpha}-\eta_{2}^{\alpha}\right)+\Gamma(\alpha+1)\left(\zeta_{2}^{\alpha+1}-\eta_{2}^{\alpha+1}\right)\right] \approx 0.5313<1 .
\end{aligned}
$$

Thus all the conditions of Theorem 3.1 hold. Therefore, the problem (4.1) has a unique solution $y_{0}(t) \in X$. Meanwhile, the problem (4.1) is Ulam-Hyers stable.

\section{Conclusions}

As a useful mathematical model, the multiple point boundary value problem of fractionalorder differential equation is used to describe many phenomena and processes such as in blood flow, chemical engineering, thermo-elasticity, underground water flow, population dynamics, and so on. The existence and stability of the solution of this kind of problem is very important in theoretical research and practical application. Therefore, we mainly study the existence and Ulam-Hyers stability of the solution of problem (1.1) in this paper. Some novel and useful criteria have been obtained by the Banach contraction principle and direct analysis methods.

\section{Acknowledgements}

The authors would like to thank the anonymous referees for their useful and valuable suggestions.

\section{Funding}

This work was supported by the National Natural Sciences Foundation of Peoples Republic of China under Grant (Nos.

$11161025 ; 11661047)$

Availability of data and materials

Not applicable.

Competing interests

The authors declare that they have no competing interests.

Authors' contributions

The authors declare that the study was realized in collaboration with equal responsibility. All authors read and approved the final manuscript.

\section{Publisher's Note}

Springer Nature remains neutral with regard to jurisdictional claims in published maps and institutional affiliations.

Received: 10 August 2020 Accepted: 28 December 2020 Published online: 11 January 2021

\section{References}

1. Shah, K., Khalil, H., Khan, R.: Investigation of positive solution to a coupled system of impulsive boundary value problems for nonlinear fractional order differential equations. Chaos Solitons Fractals 78, 329-330 (2015)

2. Ahmad, N., Ali, Z., Shah, K., Zada, A., Rahman, G.: Analysis of implicit type nonlinear dynamical problem of impulsive fractional differential equations. Complexity 2018, Article ID 6423974 (2018)

3. Ullah, A., Shah, K., Abdeljawad, T., Khan, R., Mahariq, I.: Study of impulsive fractional differential equation under Robin boundary conditions by topological degree method. Bound. Value Probl. 2020, 98 (2020)

4. Shah, K., Sher, M., Abdeljawad, T.: Study of evolution problem under Mittag-Leffler type fractional order derivative. Alex. Eng. J. 59, 3945-3951 (2020) 
5. Zhou, Y.: Attractivity for fractional differential equations in Banach space. Appl. Math. Lett. 75, 1-6 (2018)

6. Zhou, Y: Attractivity for fractional evolution equations with almost sectorial operators. Fract. Calc. Appl. Anal. 21(3), 786-800 (2018)

7. Ulam, S.: A Collection of Mathematical Problems. Interscience Tracts in Pure and Applied Mathematics, vol. 8. Interscience, New York (1906)

8. Hyers, D.: On the stability of the linear functional equation. Proc. Natl. Acad. Sci. 27, 222-2240 (1941)

9. Gorenflo, R., Kilbas, A., Mainardi, F., Rogosin, S.: Mittag-Leffler Functions, Related Topic and Applications. Springer, Berlin (2014)

10. Gao, Z., Yu, X.: Stability of nonlocal fractional Langevin differential equations involving fractional integrals. J. Appl. Math. Comput. 53, 599-611 (2017)

11. Rezaei, H., Jung, S., Rassias, T.: Laplace transform and Hyers-Ulam stability of linear differential equations. J. Math. Anal. Appl. 403, 244-251 (2013)

12. Wang, $\mathrm{C} ., \mathrm{Xu}, \mathrm{T}$.: Hyers-Ulam stability of fractional linear differential equations involving Caputo fractional derivatives. Appl. Math. Comput. 60, 383-393 (2015)

13. Agarwal, R., O'Regan, D., Hristova, S.: Stability of Caputo fractional differential equations by Lyapunov functions. Appl. Math. Comput. 60, 653-676 (2015)

14. Haq, F., Shah, K., Rahman, G.: Hyers-Ulam stability to a class of fractional differential equations with boundary conditions. Int. J. Appl. Comput. Math. 3, 1135-1147 (2017)

15. Li, C., Zhang, F.: A survey on the stability of fractional differential equations. Eur. Phys. J. Spec. Top. 193, 27-47 (2011)

16. Ibrahim, R.: Ulam-Hyers stability for Cauchy fractional differential equation in the unit disk. Abstr. Appl. Anal. 2012, $933(2012)$

17. Wang, J., Li, X.: $E_{\alpha}-$ Ulam type stability of fractional order ordinary differential equations. J. Appl. Math. Comput. 45 , 449-459 (2014)

18. Wang, J., Zhou, Y.: Ulam's type stability of impulsive ordinary differential equations. J. Math. Anal. Appl. 395, 258-264 (2012)

19. Ibrahim, R.: Generalized Ulam-Hyers stability for fractional differential equations. Int. J. Math. 23, 1250056 (2014)

20. Jiang, J., Cao, D., Chen, H.: The fixed point approach to the stability of fractional differential equations with causal operators. Qual. Theory Dyn. Syst. 15, 3-18 (2016)

21. Shah, K., Ali, A., Bushnaq, S.: Hyers-Ulam stability analysis to implicit Cauchy problem of fractional differential equations with impulsive conditions. Math. Methods Appl. Sci. 41(17), 8329-8343 (2018)

22. Wang, J., Shah, K., Ali, A.: Existence and Hyers-Ulam stability of fractional nonlinear impulsive switched coupled evolution equations. Math. Methods Appl. Sci. 41(6), 2392-2402 (2018)

23. Diaz, J., Margolis, B.: A fixed point theorem of alternative, for contractions on a generalized complete metric space. Bull. Am. Math. Soc. 74, 305-309 (1968)

24. $\mathrm{Yu}, \mathrm{X}$.: Existence and $\beta$-Ulam-Hyers stability for a class of fractional differential equations with non-instantaneous impulses. Adv. Differ. Equ. 2015, 104 (2015)

25. Fečkan, M., Wang, J., Zhou, Y.: Presentation of solutions of impulsive fractional Langevin equations and existence results. Eur. Phys. J. Spec. Top. 222, 1857-1874 (2013)

26. Zada, A., Ali, S.: Stability analysis of multi-point boundary value problem for sequential fractional differential equations with non-instantaneous impulses. Int. J. Nonlinear Sci. Numer. Simul. 19(7-8), 763-774 (2018)

27. Wang, J., Zhou, Y., Lin, Z: On a new class of impulsive fractional differential equations. Appl. Math. Comput. 242, 649-657 (2014)

28. Abbas, S., Benchohra, M.: Uniqueness and Ulam stabilities results for partial fractional differential equations with not instantaneous impulses. Appl. Math. Comput. 257, 190-198 (2015)

29. Li, H., Kao, Y:: Mittag-Leffler stability for a new coupled system of fractional-order differential equations with impulses. Appl. Math. Comput. 361, 22-31 (2019)

30. Wang, J., Zhou, Y.: Nonlinear impulsive problems for fractional differential equations and Ulam stability. Comput. Math. Appl. 64, 3389-3405 (2012)

31. Zada, A., Ali, S., Li, Y.: Ulam-type stability for a class of implicit fractional differential equations with non-instantaneous integral impulses and boundary condition. Adv. Differ. Equ. 2017, 317 (2017)

32. Kilbas, A., Srivastava, H., Trujillo, J.: Theory and Applications of Fractional Differential Equations. North-Holland Mathematics Studies, vol. 204. Elsevier, Amsterdam (2006)

\section{Submit your manuscript to a SpringerOpen ${ }^{\circ}$ journal and benefit from:}

- Convenient online submission

- Rigorous peer review

- Open access: articles freely available online

- High visibility within the field

- Retaining the copyright to your article

Submit your next manuscript at $\gg$ springeropen.com 